\title{
Electronic and Thermoelectric Properties of Transition Metal Dichalcogenides
}

\author{
Daniel I. Bilc*, Diana Benea, Viorel Pop \\ Faculty of Physics, Babeş-Bolyai University, 1 Kogălniceanu, RO-400084 Cluj-Napoca, Romania and \\ *E-mail: Daniel.Bilc@ubbcluj.ro \\ Philippe Ghosez \\ Theoretical Materials Physics, Q-MAT, CESAM, \\ Université de Liège (B5), B-4000 Liège, Belgium \\ Matthieu J. Verstraete \\ Nanomat, Q-Mat, CESAM, and European Theoretical Spectroscopy Facility, \\ Université de Liège (B5), B-4000 Liège, Belgium
}

\section{ELECTRONIC SUPPORTING INFORMATION}

\subsection{Technical Details}

The structural, electronic and thermoelectric (TE) properties were studied within B1-WC1 hybrid functional using the linear combination of atomic orbitals method and localized Gaussian basis sets as implemented in CRYSTAL ${ }^{2]}$ To study the dependence of the electronic band structure on layer thickness (quantum confinement) and epitaxial strain it is important that the structural (lattice parameters $a, c$ and $c / a$ ratio) and electronic (band gaps $\mathrm{E}_{g}$ ) properties to be described as accurately as possible. For this purpose, the exponents of the most diffuse Gaussian basis functions were optimized within B1-WC. The optimized exponents are: 0.3183 and 0.3172 for $5 \mathrm{sp}$ and $5 \mathrm{~d}$ functions of $\mathrm{Zr}$; 0.2597, 0.091, and 0.2246 for $5 \mathrm{sp}, 6 \mathrm{sp}$, and $4 \mathrm{~d}$ functions of $\mathrm{S} ; 1.5963,0.7686,0.3199$, $0.1,1.5136,1.0497$, and 0.2607 for $4 \mathrm{~s}, 4 \mathrm{p}, 5 \mathrm{sp}, 6 \mathrm{sp}, 4 \mathrm{~d}$, $5 \mathrm{~d}$, and $6 \mathrm{~d}$ functions of Se; 2.3841, 0.5058, 0.292, and 0.1298 for $5 \mathrm{sp}, 6 \mathrm{sp}, 5 \mathrm{~d}$ and $6 \mathrm{~d}$ functions of Mo; 0.5860 and 0.1146 for $7 \mathrm{sp}$ and $6 \mathrm{~d}$ functions of $\mathrm{W} ; 0.828,0.1531$, and 0.1184 for $6 \mathrm{sp}, 7 \mathrm{sp}$ and $6 \mathrm{~d}$ functions of Hf; 2.5335, $0.2056,0.8050$, and 0.2259 for $5 \mathrm{p}, 6 \mathrm{sp}, 5 \mathrm{~d}$, and $6 \mathrm{~d}$ functions of Sn; 1.8395, 0.5048, 0.1643, 1.2672, 0.4947, and 0.1427 for $6 \mathrm{~s}, 6 \mathrm{sp}, 7 \mathrm{sp}, 5 \mathrm{~d}, 6 \mathrm{~d}$, and $7 \mathrm{~d}$ functions of Te. To increase the efficiency of CRYSTAL code, the two outermost $\mathrm{s}$ and $\mathrm{p}$ functions of Se were combined into $5 \mathrm{sp}$ and $6 \mathrm{sp}$ functions, the $6 \mathrm{~s}$, and $6 \mathrm{p}$ functions of Sn were combined into a $6 \mathrm{sp}$ function, the 5211 contraction pattern of Te s functions was changed into a 5221 contraction and the most two outer $\mathrm{s}$ and $\mathrm{p}$ functions were combined into $6 \mathrm{sp}$ and $7 \mathrm{sp}$ functions, the 2111 contraction pattern of Mo s functions was changed into a 221 contraction having the $5 \mathrm{sp}$ and $6 \mathrm{sp}$ combined functions and the $4 \mathrm{f}$ polarization function was removed. $\mathrm{B} 1-\mathrm{WC}^{1}$ mixes the generalized gradient approximation functional of $\mathrm{Wu}$ and Cohen ${ }^{[3}$ with $16 \%$ of exact exchange within $\mathrm{B} 1^{4}$ scheme. $\mathrm{B} 1-\mathrm{WC}$ is more appropriate to treat $d$ and $f$ electronic materials, being a good alternative to $\mathrm{LDA}(\mathrm{GGA})+\mathrm{U}^{5[6}$

\subsection{Effect of Spin-Orbit Interaction}

In order to check the effect of spin-orbit interactions (SOI) on the electronic and TE properties of high performance TMDs, we used also the augmented plane wave and local orbital (APW + lo) method as implemented in Wien $2 \mathrm{k}$ code ${ }^{7}$ These calculations were performed within the partial implementation of B1-WC hybrid functional. In Wien $2 \mathrm{k}$ and APW + lo method the hybrid functionals are implemented only inside of augmented-plane-wave spheres around the atoms. These radii were taken to be 2.4 Bohr, 2.3 Bohr and 2.5 Bohr for W, Se and Sn atoms, respectively. The scalar relativistic effects were also included. The self-consistent iterations were performed for the optimized B1-WC lattice constants and atomic positions using $6 \times 6 \times 6 \mathrm{k}$-point meshes inside of the Brillouin zone to within $0.0001 \mathrm{Ry}$. The number of plane waves is defined by $\mathrm{RK}_{\max }$ whereas the largest vector in the charge-density Fourier expansion is defined by $\mathrm{G}_{\max }$, which were taken to be 9 and 16, respectively. The transport properties were estimated using very dense $97 \times 97 \times 67$ and $67 \times 67 \times 47 k$-point meshes for bulk and monolayer (1ML) structures, respectively. The relaxation time $\tau_{a}=1 \times 10^{-14} \mathrm{~s}$ in $a$ direction was used in the study of TE properties.

The electronic band structures with and without SOI for $1 \mathrm{ML}$ and bulk structures of $\mathrm{WSe}_{2}$ and $\mathrm{SnSe}_{2}$ are shown in Figures $\mathrm{S} 1$ and $\mathrm{S} 2$. SOI has the most dominant effect on the electronic band structure of $\mathrm{WSe}_{2} 1 \mathrm{ML}$ by spitting the highest valence bands along $\Gamma K$ direction with a splitting value of $\sim 0.4 \mathrm{eV}$ at $\mathrm{K}$ point, and the lowest conduction bands near the $\Lambda$ minimum along $\Gamma K$ direction (Fig. S1 a, b). For $\mathrm{WSe}_{2}$ bulk, SOI splits the lowest conduction bands and highest valence bands along $\mathrm{AH}$ direction with a splitting value of $\sim 0.4 \mathrm{eV}$ at $\mathrm{H}$ point in the valence band (Fig. S1k, d). SOI decreases the direct band gap of $\mathrm{WSe}_{2} 1 \mathrm{ML}$ from $\sim 1.4 \mathrm{eV}$ to $\sim 1.27 \mathrm{eV}$, whereas the indirect band gap of $\mathrm{WSe}_{2}$ bulk from $\sim 0.8$ $\mathrm{eV}$ to $\sim 0.73 \mathrm{eV}$. In the case of $\mathrm{SnSe}_{2} 1 \mathrm{ML}$ and bulk, SOI splits the highest valence bands along $\Gamma \mathrm{A}$ direction by $\sim 0.27 \mathrm{eV}$ and avoids the band crossing along $\mathrm{MK}(\mathrm{LH})$ and $\Gamma M$ directions (Fig. S2 a-d). The indirect band gap of $\mathrm{SnSe}_{2}$ bulk is decreased by SOI from $\sim 0.2 \mathrm{eV}$ to $\sim 0.17$ 

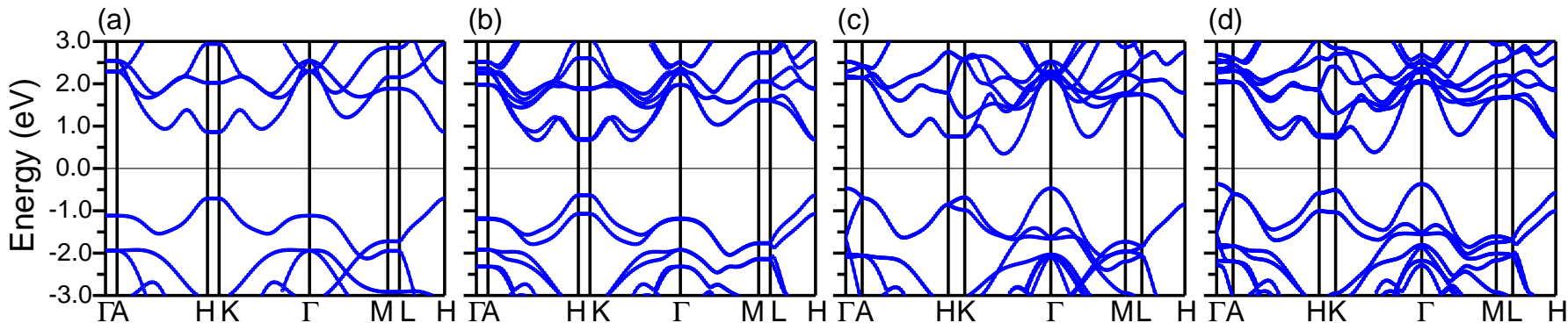

Figure S1: Effect of spin-orbit interaction (SOI) on electronic band structure of $\mathrm{WSe}_{2}$ monolayer (1ML) and bulk: (a) WSe $1 \mathrm{ML}$ without SOI, (b) WSe 2 1ML with SOI, (c) $\mathrm{WSe}_{2}$ bulk without SOI, and (d) WSe $\mathrm{Wulk}_{2}$ with SOI estimated within B1-WC partial hybrid implementation in Wien2k code.
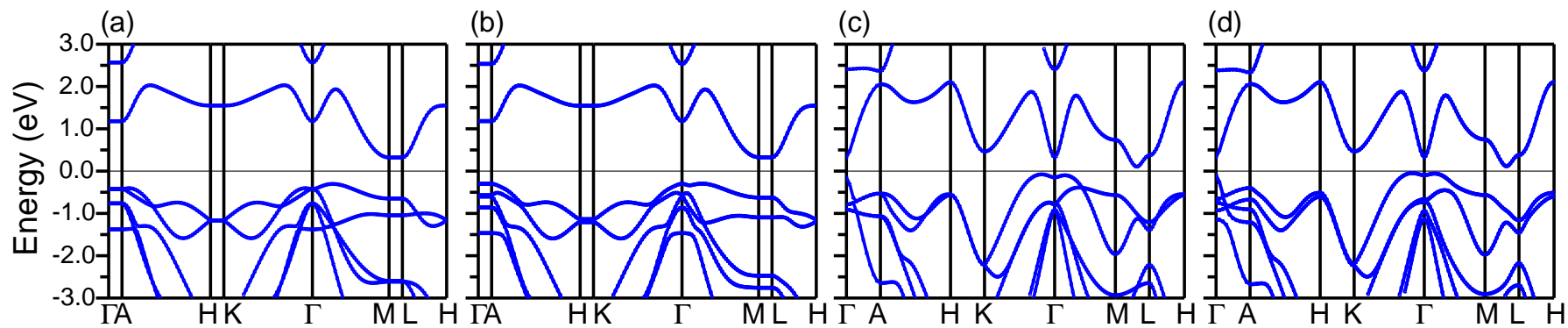

Figure S2: Effect of spin-orbit interaction (SOI) on electronic band structure of $\mathrm{SnSe}_{2}$ monolayer (1ML) and bulk: (a) $\mathrm{SnSe}_{2}$ $1 \mathrm{ML}$ without SOI, (b) $\mathrm{SnSe}_{2} 1 \mathrm{ML}$ with SOI, (c) $\mathrm{SnSe}_{2}$ bulk without SOI, and (d) $\mathrm{SnSe}_{2}$ bulk with SOI estimated within B1-WC partial hybrid implementation in Wien2k code.
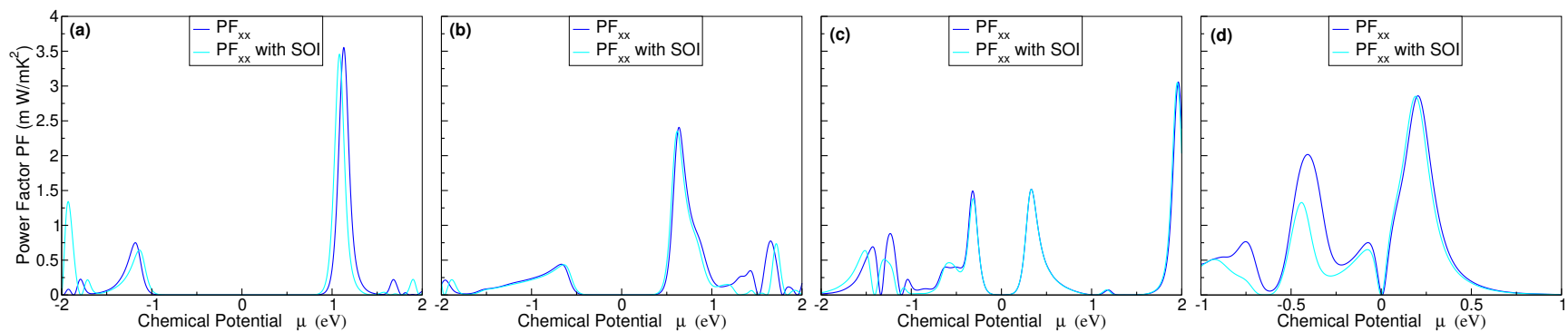

Figure S3: Power factor $\mathrm{PF}_{x x}$ dependence on chemical potential for: (a) WSe $\mathrm{W}_{2}$ monolayer (1ML), (b) WSe 2 bulk, (c) SnSe 2 $1 \mathrm{ML}$, and (d) $\mathrm{SnSe}_{2}$ bulk, estimated within B1-WC partial hybrid without/with spin-orbit interaction (SOI) at $300 \mathrm{~K}$ using the relaxation time $\tau_{a}=1 \times 10^{-14} \mathrm{~s}$.

$\mathrm{eV}$.

$\mathrm{PF}_{x x}$ along $a$ direction estimated with and without SOI within B1-WC partial hybrid functional for 1ML and bulk structures of $\mathrm{WSe}_{2}$ and $\mathrm{SnSe}_{2}$ is shown in Figure S3. The effect of SOI on maximum $\mathrm{PF}_{x x}$ is not significant at the optimal carrier concentration doping which can be achieved experimentally and when the chemical potential lays at $\sim 1.2 \mathrm{eV}(\sim-1.2 \mathrm{eV}), \sim 0.7 \mathrm{eV}(\sim-0.7 \mathrm{eV}), \sim 0.3$ $\mathrm{eV}(\sim-0.3 \mathrm{eV})$, and $\sim 0.2 \mathrm{eV}(\sim-0.1 \mathrm{eV})$ for n-type (ptype) doping of $\mathrm{WSe}_{2} 1 \mathrm{ML}, \mathrm{WSe}_{2}$ bulk, $\mathrm{SnSe}_{2} 1 \mathrm{ML}$, and $\mathrm{SnSe}_{2}$ bulk, respectively. Since $\mathrm{PF}_{x x}$ at optimal doping are not significantly affected by SOI and because SOI can not be treated within B1-WC hybrid implementation of CRYSTAL code, the electronic and TE properties of TMDs were studied without the inclusion of SOI. 

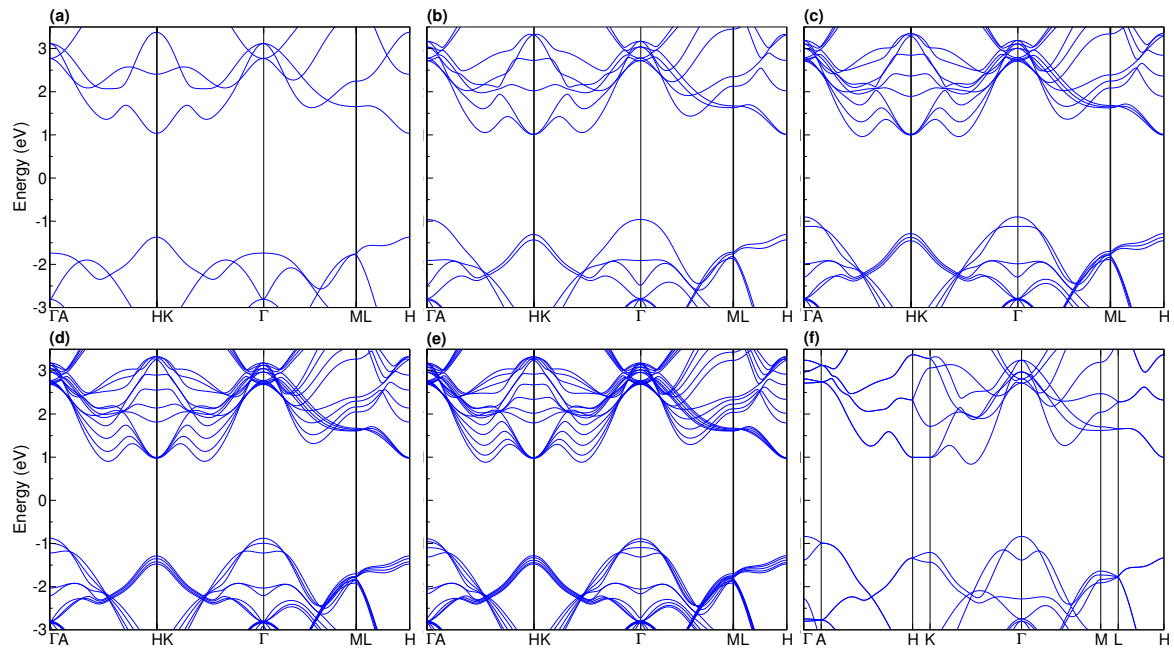

Figure S4: Electronic band structure of $\mathrm{WSe}_{2}$ monolayers (MLs): (a) 1ML, (b) 2MLs, (c) 3MLs, (d) 4MLs, (e) 5MLs, and (f) $\mathrm{WSe}_{2}$ bulk estimated within B1-WC.
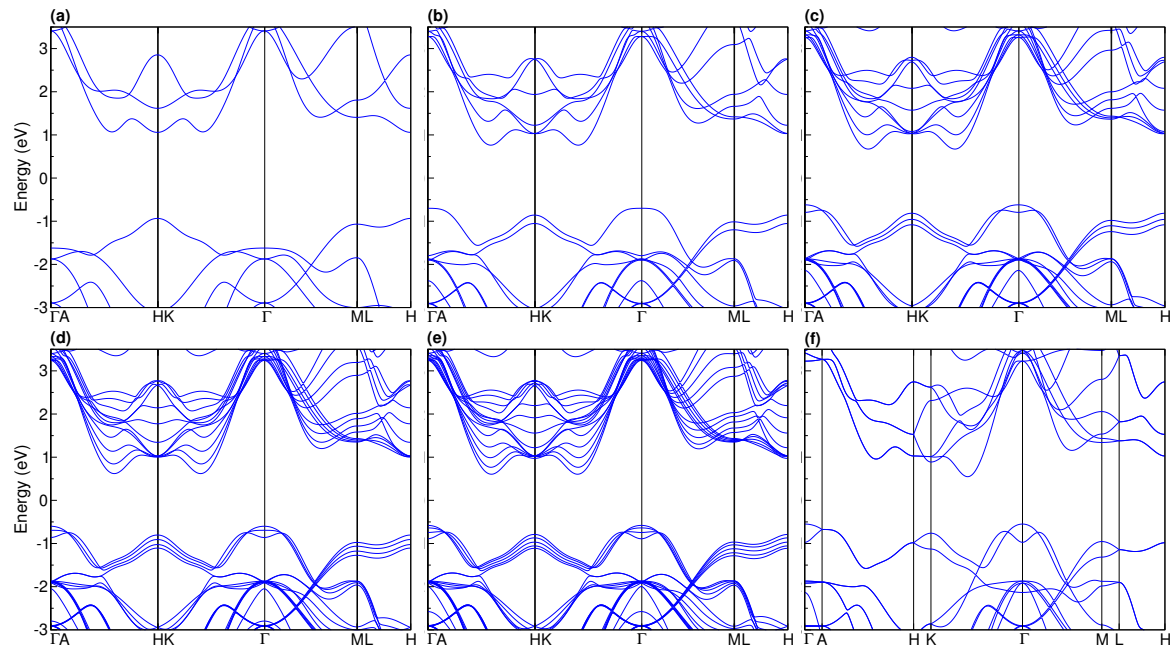

Figure S5: Electronic band structure of $\mathrm{MoTe}_{2}$ monolayers (MLs): (a) 1ML, (b) 2MLs, (c) 3MLs, (d) 4MLs, (e) 5MLs, and (f) $\mathrm{MoTe}_{2}$ bulk estimated within B1-WC.

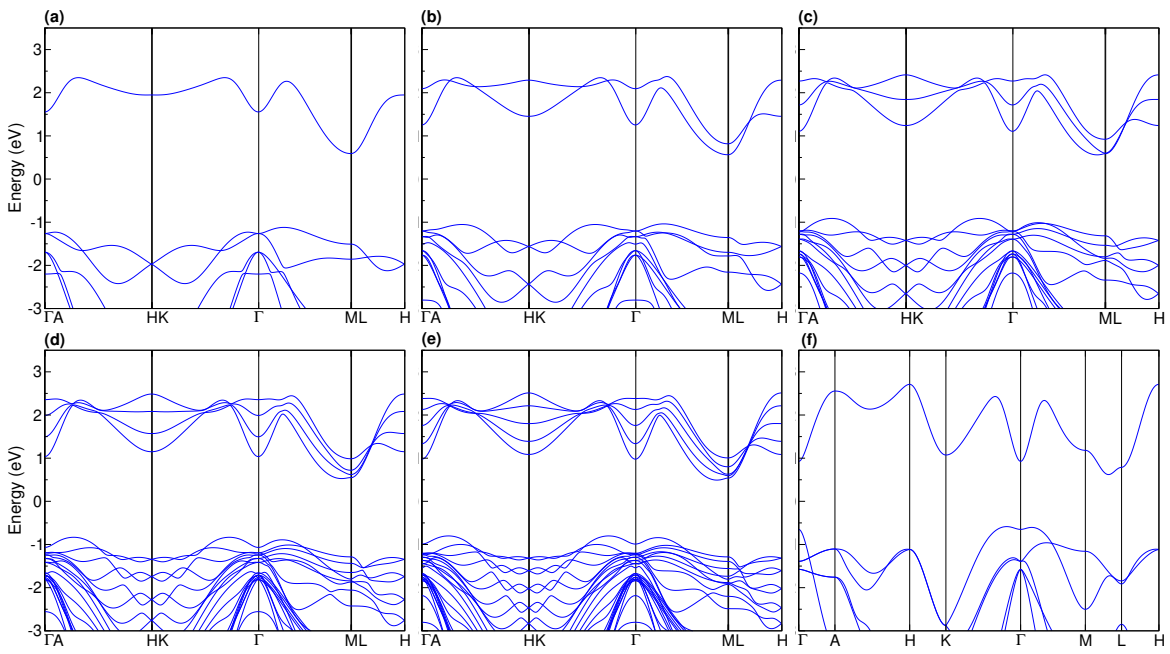

Figure S6: Electronic band structure of $\mathrm{SnSe}_{2}$ monolayers (MLs): (a) 1ML, (b) 2MLs, (c) 3MLs, (d) 4MLs, (e) 5MLs, and (f) $\mathrm{SnSe}_{2}$ bulk estimated within B1-WC. 


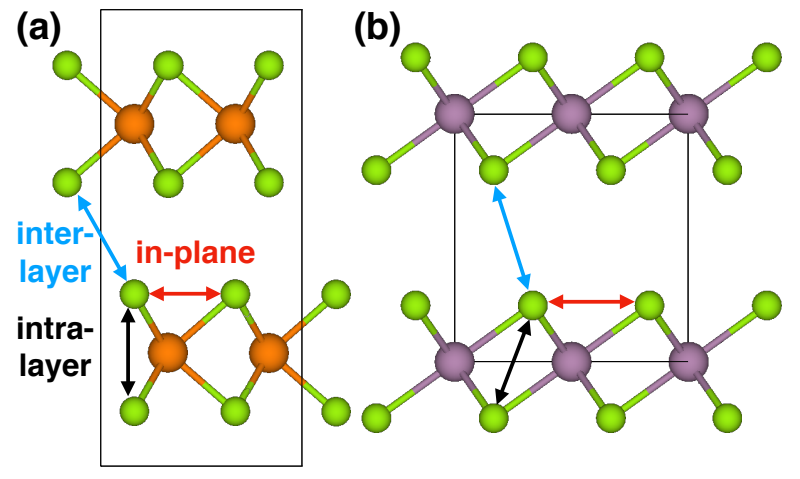

Figure S7: The different Se-Se atomic interactions for the $2 \times 2 \times 1$ supercells of: (a) $2 \mathrm{H}$ and (b) $1 \mathrm{~T}$ structures viewed in bc plane.

\subsection{Bonding Information Analysis}

Crystal Orbital Hamilton Population (COHP) method was used to extract the bonding information for different pairs of atoms and their orbitals contribution. $\frac{8}{}$ The different intra-layer, inter-layer and in-plane Se-Se atomic interactions are represented in Figure $\mathrm{S} 7$ for the $2 \times 2 \times 1$ supercells of $2 \mathrm{H}$ and $1 \mathrm{~T}$ structures viewed in $b c$ plane. Figure S8 shows COHP for M-Se and Se-Se intra-layer bonding, Se-Se inter-layer bonding (van der Waals), SeSe and M-M in-plane bonding through the $s p^{2}$ hybridization in $a b$ plane ( $\mathrm{M}$ - transition metal, Sn). The high- est top VB states of $\mathrm{WSe}_{2}$ in the $[-0.83 \mathrm{eV},-1 \mathrm{eV}]$ energy range have (i) $p_{z}$ contribution from very small $\sigma$ intralayer, and $\pi$ in-plane Se-Se bonding, (ii) $p$ contribution from very small $\pi^{*}$ inter-layer Se-Se antibonding, and (iii) $d_{z^{2}}$ contribution from very small $\pi$ in-plane W-W bonding. In the $[-1 \mathrm{eV},-1.4 \mathrm{eV}]$ energy range, the electronic charge from $p_{z}$ orbitals of Se is displaced along Se-Se in-plane direction making at the same time $\pi$ inplane Se-Se bonding, $\pi^{*}$ intra- and $\pi^{*}$ inter-layer Se-Se antibonding. The top VB states in the $[-0.83 \mathrm{eV},-1.4 \mathrm{eV}]$ energy range have nonbonding character form $\mathrm{W}$-Se interactions inside atomic layers. The lowest CB states in the $[0.83 \mathrm{eV}, 1 \mathrm{eV}]$ energy range have $s, p_{x}$ and $p_{y}$ contributions from $\pi$ intra-layer Se-Se bonding and $\sigma^{*}$ in-plane Se-Se antibonding, and antibonding character form WSe interactions. CB states in $[1 \mathrm{eV}, 1.4 \mathrm{eV}]$ energy range associated to the very anisotropic band along HK direction have the dominant $d_{z^{2}}$ contribution from $\pi^{*}$ in-plane $\mathrm{W}-\mathrm{W}$ antibonding (Figure S8p). The highest top VB states of $\mathrm{WSe}_{2} 1 \mathrm{ML}$ laying in the $[-1.37 \mathrm{eV},-1.5 \mathrm{eV}]$ energy range have contribution from $\sigma^{*}$ in-plane $\mathrm{W}-\mathrm{W}$ antibonding and $\mathrm{W}$-Se antibonding, whereas the lowest $\mathrm{CB}$ states laying in $[1 \mathrm{eV}, 1.3 \mathrm{eV}]$ energy range have contribution from $\pi^{*}$ in-plane W-W antibonding and W-Se antibonding. The electronic charge describing in-plane and intra-layer Se-Se interactions of $\mathrm{WSe}_{2} 1 \mathrm{ML}$ is localized deeper in energy, bellow $-1.5 \mathrm{eV}$ in $\mathrm{VB}$ and above $1.3 \mathrm{eV}$ in $\mathrm{CB}$ (Figure $\mathrm{S} 8 \mathrm{a}$ ).
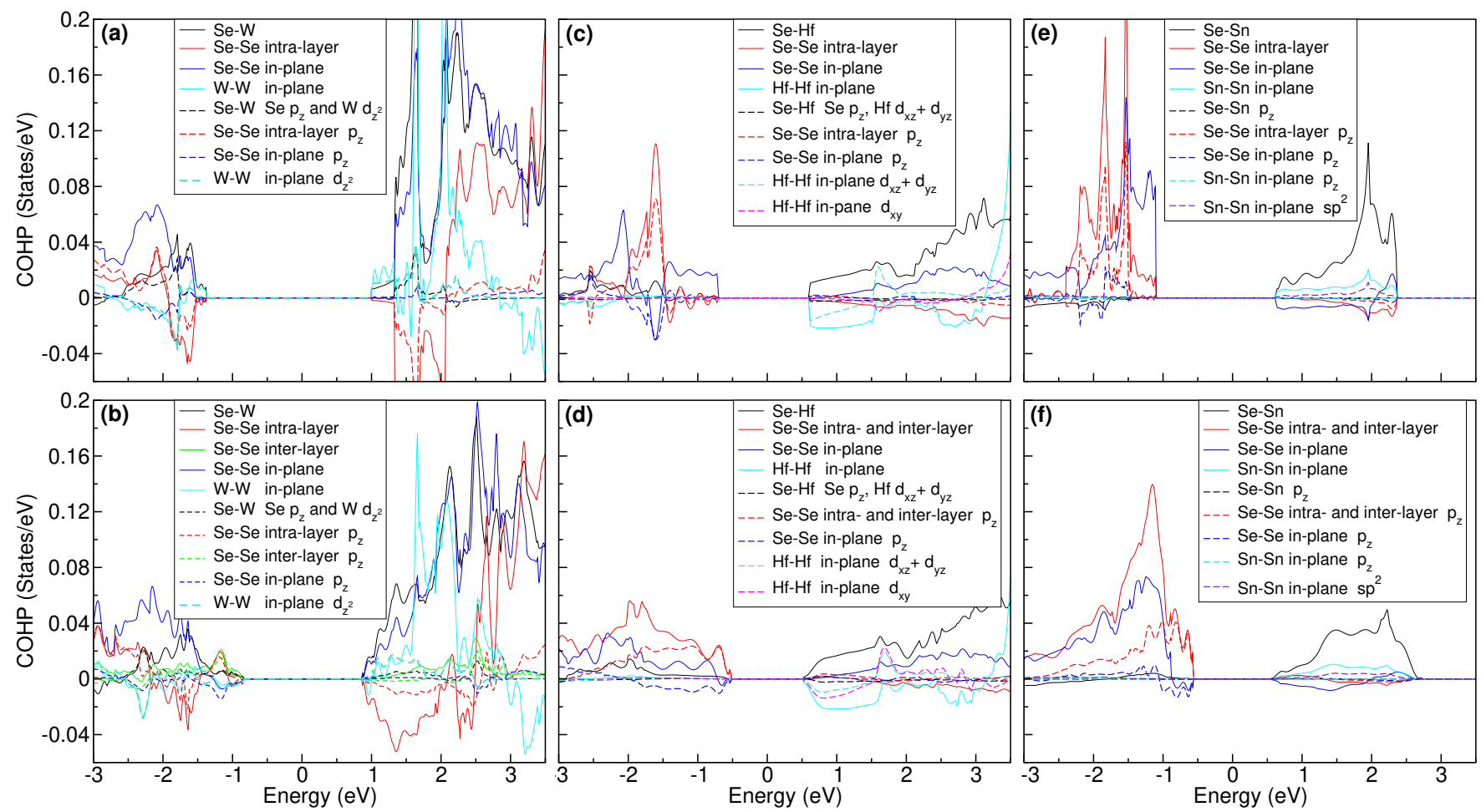

Figure S8: Crystal Orbital Hamilton Population (COHP) method to extract bonding information for different pairs of atoms and their orbitals contribution in: (a) WSe 2 monolayer (ML), (b) WSe $\mathrm{W}_{2}$ bulk, (c) HfSe $\mathrm{HL}_{2} \mathrm{ML}$ (d) HfSe $\mathrm{H}_{2}$ bulk, (e) SnSe $\mathrm{SL}_{2}$, and

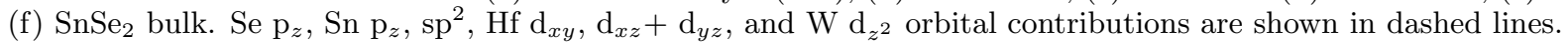


The top VB states of $\mathrm{HfSe}_{2}$ in the $[-0.51 \mathrm{eV},-1 \mathrm{eV}]$ energy range have (i) $p_{z}$ contribution from $\pi^{*}$ inter-layer Se-Se antibonding and $\pi$ in-plane Se-Se bonding, (ii) $s$, $p_{x}$ and $p_{y}$ contributions from $\sigma^{*}$ in-plane Se-Se antibonding, and (iii) nonbonding character for Hf-Se interactions. The bottom of $\mathrm{CB}$ states in the $[0.5 \mathrm{eV}, 1 \mathrm{eV}]$ energy range have (i) $d_{x y}$ contribution from $\sigma$ in-plane Hf-Hf bonding, (ii) $d_{x z}$, and $d_{y z}$ contributions from $\pi$ in-plane Hf-Hf bonding, and (iii) antibonding character form Hf-Se interactions (Figure $\mathrm{S} 8 \mathrm{~d}$ ). For $\mathrm{HfSe}_{2} 1 \mathrm{ML}, p_{z}$ contribution to VB states from $\pi^{*}$ intra-layer Se-Se antibonding and $\pi$ in-plane Se-Se bonding is shifted down in energy bellow $-1.5 \mathrm{eV}$, and the top VB states have only $s, p_{x}$ and $p_{y}$ contributions from $\sigma^{*}$ in-plane Se-Se antibonding. Also $d_{x y}$ contribution from $\sigma^{*}$ in-plane Hf-Hf antibonding is shifted up in energy above $3 \mathrm{eV}$ in $\mathrm{CB}$, such that the bottom of CB states have $d_{x z}$, and $d_{y z}$ contributions from $\pi$ in-plane Hf-Hf bonding, and antibonding character form Hf-Se interactions (Figure S8.).

The highest top VB states of $\mathrm{SnSe}_{2}$ in the $[-0.6 \mathrm{eV}$,$0.9 \mathrm{eV}]$ energy range have $p_{z}$ contribution from $\pi^{*}$ intraand inter-layer Se-Se antibonding, and small $\pi$ in-plane Se-Se bonding. The electronic charge associated to $\sigma^{*}$ inplane Se-Se antibonding is localized bellow $-0.9 \mathrm{eV}$ in VB. The CB states of $\mathrm{SnSe}_{2}$ in the $[0.6 \mathrm{eV}, 1.2 \mathrm{eV}]$ energy range have contributions from $\sigma$ in-plane Se-Se bonding, small $\pi^{*}$ in-plane Sn-Sn antibonding, and antibonding character form Sn-Se interactions (Figure $\mathrm{S} 8$ ). For $\mathrm{SnSe}_{2}$ $1 \mathrm{ML}, p_{z}$ contribution to VB states from $\pi^{*}$ intra-layer and in-plane Se-Se antibonding is shifted down in energy bellow $-1.5 \mathrm{eV}$, and the contributions to bottom of $\mathrm{CB}$ states in the $[0.6 \mathrm{eV}, 1.2 \mathrm{eV}]$ energy range from $\pi^{*}$ in-plane Sn-Sn antibonding, $\sigma$ in-plane Se-Se bonding, and Sn-Se antibonding are slightly larger compared to $\mathrm{SnSe}_{2}$ bulk (Figure S8, f).

\subsection{Electronic band anisotropy and effective mass quality factors}

It is desired to know what type of electronic band structure is able to maximize TE performance $(Z T)$ at a given carrier concentration $n$ and $T$, which define the position of chemical potential $\mu$. More exactly, we look at the effect of electronic band multiplicity/degeneracy $N_{v}$ and band anisotropy on TE performance at a given fixed $\mu$ value. For anisotropic electronic bands, the energy dispersion relative to band minima is given by:

$$
\epsilon_{\vec{k}}=\frac{\hbar^{2}}{2}\left(\frac{1}{m_{l}} k_{l}^{2}+\frac{2}{m_{t}} k_{t}^{2}\right)
$$

where $m_{l}$ and $m_{t}$ are the effective masses along the longitudinal and transverse directions of the carrier pockets with ellipsoidal Fermi surfaces. The elipsoidal carrier pockets can be converted to a pocket model with spheri- cal shape as:

$$
\begin{aligned}
k_{l}^{2} & =\frac{m_{l}}{m_{d}^{\prime}}{k^{\prime}}_{l}^{2} \\
k_{t}^{2} & =\frac{m_{t}}{m^{\prime} d}{k^{\prime}}_{t}^{2} \\
\epsilon_{k^{\prime}}=\frac{\hbar^{2}}{2 m_{d}^{\prime}}\left({k^{\prime}}_{l}^{2}+2{k^{\prime}}_{t}^{2}\right) & =\frac{\hbar^{2}}{2 m_{d}^{\prime}} k^{\prime 2}
\end{aligned}
$$

where $m^{\prime}{ }_{d}=\left(m_{l} m_{t}^{2}\right)^{1 / 3}$ relation is given by setting the equality between the infinitesimal volumes $d^{3} \vec{k}=d^{3} \overrightarrow{k^{\prime}}$. Considering the spin 2 and band $N_{v}$ degeneracies and the ocupation of electronic states in inverse space $\overrightarrow{k^{\prime}}$ given by Fermi distribution function, the concentration of carriers $n$ can be written as:

$$
\begin{aligned}
& n=\frac{2 N_{v}}{(2 \pi)^{3}} \int \frac{d^{3} \overrightarrow{k^{\prime}}}{\exp \left(\frac{\epsilon_{\overrightarrow{k^{\prime}}}-\mu}{K_{B} T}\right)+1} \\
& n=\frac{2^{1 / 2}}{\pi^{2} \hbar^{3}} N_{v} m^{\prime}{ }_{d}^{3 / 2} \int_{0}^{\infty} \frac{\epsilon^{1 / 2} d \epsilon}{\exp \left(\frac{\epsilon-\mu}{K_{B} T}\right)+1}
\end{aligned}
$$

whereas for a single spherical pocket with the energy dispersion $\epsilon_{\vec{k}}=\hbar^{2} k^{2} /\left(2 m_{d}\right), n$ is given by:

$$
n=\frac{2^{1 / 2}}{\pi^{2} \hbar^{3}} m_{d}^{3 / 2} \int_{0}^{\infty} \frac{\epsilon^{1 / 2} d \epsilon}{\exp \left(\frac{\epsilon-\mu}{K_{B} T}\right)+1}
$$

From Eqs. 6 and 7, the maximization of TE performance at given $n, T$, and $\mu$ constant values, implies also constant effective mass values $m_{d}{ }^{3 / 2}=N_{v} m_{d}^{\prime}{ }^{3 / 2}$, where $m_{d}$ is called density of states effective mass $\left(m_{d}=\right.$ $\left.N_{v}{ }^{2 / 3}\left(m_{l} m_{t}^{2}\right)^{1 / 3}\right)$. Therefore, the more appropriate question is what type of electronic band structure is able to maximize TE performance for a given constant $m_{d}$ value.

It was shown that $Z T$ increases with $B$ quality factor given by ${ }^{9}-11$ :

$$
\begin{array}{r}
B=\frac{1}{3 \pi^{2}}\left(\frac{2 K_{B} T}{\hbar^{2}}\right)^{3 / 2} m_{d}{ }^{3 / 2} \frac{K_{B}{ }^{2} \hat{\mu} T}{e \kappa_{l}}=\frac{B_{E} T}{\kappa_{l}} \\
B_{E}=\frac{1}{3 \pi^{2}}\left(\frac{2 K_{B} T}{\hbar^{2}}\right)^{3 / 2} m_{d}{ }^{3 / 2} \frac{K_{B}{ }^{2} \hat{\mu}}{e}
\end{array}
$$

where $B_{E}$ is electronic quality factor, $\hat{\mu}$ is carrier mobility along transport direction, $\kappa_{l}$ is the lattice thermal conductivity, $K_{B}$ is Boltzmann constant and $e$ is electron charge. The carrier mobility $\hat{\mu}_{i i}$ and electrical conductivity $\sigma_{i i}$ along an orthogonal $i$ direction $(i=x, y, z)$ are defined as:

$$
\begin{array}{r}
\hat{\mu}_{i i}=\frac{1}{N_{v}} \sum_{j=1}^{N_{v}} \hat{\mu}_{i i}^{j}=\frac{1}{N_{v}} \sum_{j=1}^{N_{v}} \frac{e \tau_{i i}}{m_{i i}^{j}} \\
\sigma_{i i}=n e \hat{\mu}_{i i}
\end{array}
$$

where the summation over $j$ accounts for the contribution of each carrier pocket to $\hat{\mu}_{i i}, \tau_{i i}$ and $m_{i i}^{j}$ are relaxation 
time and effective mass components along $i$ direction. In the case of anisotropic ellipsoidal pockets the contribution of $m_{l}$ and $m_{t}$ to $\hat{\mu}_{i i}$ is determined by the crystal symmetry and the orientation of ellipsoidal axis relative to $i$ direction. For cubic symmetry and also for lower trigonal symmetry having the ellipsoidal pockets tilted with respect to $i$ direction, both $m_{l}$ and $m_{t}$ will enter in the expression of $\hat{\mu}_{i i}$ :

$$
\hat{\mu}_{i i}=\frac{1}{3}\left(\frac{1}{m_{l}}+\frac{2}{m_{t}}\right) e \tau_{i i}=\frac{1}{m_{I}} e \tau_{i i}
$$

where $m_{I}$ is the inertial mass or conductivity mass $m_{c}$ $\left(m_{I}=m_{c}\right)$.

At high $T$ where $Z T$ reaches its maximum value, the dominant electron-phonon scattering mechanisms are due to acoustic phonons. The relaxation time $\tau_{i i}$ for acoustic anisotropic scattering can be estimated using the deformation potential theory $\sqrt{12}[14$ as:

$$
\tau_{i i}=\frac{\pi \hbar^{4} C_{l}}{2^{1 / 2} \Xi^{2}\left(K_{B} T\right)^{3 / 2}\left(m_{l} m_{t}^{2}\right)^{1 / 2}}
$$

where $C_{l}$ is a combination of elastic constants, and $\Xi$ is deformation potential coefficient. Thus at high $T$ and even for a high band anisotropy with large anisotropy ratio $R=m_{l} / m_{t}$, the acoustic relaxation time $\tau_{i i}$ is relatively isotropic $\left(\tau_{x x} \sim \tau_{y y} \sim \tau_{z z}\right)$. From Eqs. 8, 12, and $13, B$ quality factor can be expressed as:

$$
\begin{array}{r}
B=\frac{2 \hbar K_{B}^{2} C_{l} T}{3 \pi \Xi^{2} \kappa_{l}} \frac{N_{v}}{m_{I}} \\
B=\frac{2 \hbar K_{B}^{2} C_{l} T}{3 \pi \Xi^{2} \kappa_{l} m_{d}} B_{m} \\
B_{m}=\frac{N_{v} m_{d}}{m_{I}}
\end{array}
$$

where we define the adimensional factor $B_{m}$ as effective mass quality factor, which has to be maximized for a given constant $m_{d}$ value. The ratio $m_{d} / m_{I}$ was also identified as a figure of merit for the effect of electronic band anisotropy 15 . $B_{m}$ is related to the Fermi surface complexity factor $N_{v}^{*} K^{*}$ defined as $N_{v}^{*} K^{*}=\left(m_{d} / m_{I}\right)^{3 / 2 ! 16}$ Using the relations between $m_{d}$ (resp. $m_{I}$ ) and $m_{l}$ (resp. $\left.m_{t}\right), B_{m}$ can be expressed as:

$$
B_{m}=\frac{1}{3} N_{v}^{5 / 3} R^{1 / 3}\left(2+\frac{1}{R}\right)
$$

where $R$ is the effective mass anisotropy ratio $R=$ $m_{l} / m_{t}$. Therefore, the maximization of TE performance $\left(Z T, B\right.$, and $\left.B_{m}\right)$ at a given charge carrier density $n$ and $T$ (resp. at a given $m_{d}$ ) can be achieved by increasing simultaneously the degeneracy/multiplicity $N_{v}$ of electronic bands and their anisotropy ratio $R\left(B_{m}=1\right.$ for a single isotropic carrier pocket).

In the case of highly anisotropic materials as TMDs, the ellipsoidal longitudinal $l$ axis is parallel to $c$ crystallographic direction such that $m_{l}=m_{z z}$ contributes only to carrier mobility $\hat{\mu}_{c}$ along $c$ direction $\left(\hat{\mu}_{c}=e \tau_{c} / m_{l}\right)$, whereas $m_{t}$ contributes only to carrier mobility $\hat{\mu}_{a b}$ in $a b$ plane $\left(\hat{\mu}_{a b}=e \tau_{a b} / m_{t}\right)$. The relation between transverse and orthogonal effective masses, $m_{t}=\left(m_{x x} m_{y y}\right)^{1 / 2}$, can be found by setting the equality between the infinitesimal areas $d^{2} k_{t}=d k_{x} d k_{y}$ in $a b$ plane. At high $T$ and for relatively isotropic acoustic relaxation time $\tau_{a b} \sim \tau_{c}$ described by Eq. 13, the effective mass quality factor $B_{m, a b}$ in $a b$ plane, and $B_{m, c}$ along $c$ direction are given by:

$$
\begin{aligned}
& B_{m, a b}=\frac{N_{v} m_{d}}{m_{t}}=N_{v}^{5 / 3} R^{1 / 3} \\
& B_{m, c}=\frac{N_{v} m_{d}}{m_{l}}=N_{v}^{5 / 3} R^{-2 / 3}
\end{aligned}
$$

where $R=m_{z z} /\left(m_{x x} m_{y y}\right)^{1 / 2}$ in terms of orthogonal effective masses.

In order to quantify TE performance of TMDs monolayers (MLs), we consider 2D quantum well in which the electrons are confined to move in the $a b$ plane and having the occupation energy given by the lowest quantization energy of the bound state in $z$ direction 9 :

$$
\epsilon_{\vec{k}}=\frac{\hbar^{2}}{2}\left(\frac{1}{m_{x x}} k_{x}^{2}+\frac{1}{m_{y y}} k_{y}^{2}+\frac{\pi^{2}}{m_{z z} L_{z}^{2}}\right)
$$

where $L_{z}$ is the width of quantum well along $z$ direction. The carrier density $n^{2 D}$, quality factor $B^{2 D}$, and carrier mobility $\hat{\mu}_{a b}^{2 D}$ in $a b$ plane of $2 \mathrm{D}$ quantum well can be expressed as 917 :

$$
\begin{array}{r}
n^{2 D}=\frac{1}{\pi L_{z} \hbar^{2}} N_{v}\left(m_{x x} m_{y y}\right)^{1 / 2} \int_{0}^{\infty} \frac{d \epsilon}{\exp \left(\frac{\epsilon-\mu}{K_{B} T}\right)+1} \\
B^{2 D}=\frac{K_{B}^{3} T^{2}}{\pi L_{z} \hbar^{2} e \kappa_{l}} N_{v}\left(m_{x x} m_{y y}\right)^{1 / 2} \hat{\mu}_{a b}^{2 D} \\
\hat{\mu}_{a b}^{2 D}=\frac{e \tau_{a b}^{2 D}}{\left(m_{x x} m_{y y}\right)^{1 / 2}}=\frac{2 L_{z} e \hbar^{3} C_{l}}{3 K_{B} T \Xi^{2}\left(m_{x x} m_{y y}\right)}
\end{array}
$$

where $\tau_{a b}^{2 D}$ is the acoustic relaxation time at high $T$ of $2 \mathrm{D}$ quantum well. From Eqs. 22 and $23, B^{2 D}$ is given by:

$$
\begin{gathered}
B^{2 D}=\frac{2 \hbar K_{B}^{2} C_{l} T}{3 \pi \Xi^{2} \kappa_{l}} \frac{N_{v}}{\left(m_{x x} m_{y y}\right)^{1 / 2}} \\
B^{2 D}=\frac{2 \hbar K_{B}^{2} C_{l} T}{3 \pi \Xi^{2} \kappa_{l} m_{d}^{2 D}} B_{m}^{2 D} \\
B_{m}^{2 D}=\frac{N_{v} m_{d}^{2 D}}{\left(m_{x x} m_{y y}\right)^{1 / 2}}=N_{v}^{2}
\end{gathered}
$$

where we define $B_{m}^{2 D}$ as $2 D$ effective mass quality factor which has to be maximized for a given constant density of states effective mass $m_{d}^{2 D}$ of the quantum well $\left(m_{d}^{2 D}=\right.$ $N_{v}\left(m_{x x} m_{y y}\right)^{1 / 2}=$ const $)$. Therefore at a given $m_{d}^{2 D}$, TE performance of a $2 \mathrm{D}$ quantum well $\left(Z T^{2 D}, B^{2 D}\right.$, and $B_{m}^{2 D}$ ) is proportional with $N_{v}^{2}$. 
Table S1: Effective masses $m_{i i}\left(m_{e}\right)$ (i $=\mathrm{x}$, y orthogonal directions, and $m_{e}$ is free electron mass), carrier pocket degeneracy (or band multiplicity) $N_{v}$, and $2 \mathrm{D}$ effective mass quality factor $B_{m}^{2 D}=N_{v}^{2}$ for CB minima and VB maxima of MoTe 2 1-2MLs. Effective masses $m_{i i}\left(m_{e}\right)(\mathrm{i}=\mathrm{x}, \mathrm{y}, \mathrm{z})$, carrier pocket degeneracy $N_{v}$, anisotropy ratio $R=m_{z z} /\left(m_{x x} m_{y y}\right)^{1 / 2}$, and effective mass quality factor $B_{m, a b}=N_{v}^{5 / 3} R^{1 / 3}$ in $a b$ plane, for CB minima and VB maxima of MoTe 2 strained and unstrained bulk. The extremum points are specified in fraction of primitive inverse space vectors $k_{j}(\mathrm{j}=1,2,3)$ and their localization in eV are relative to $\mathrm{CB}$ minimum and VB maximum.

\begin{tabular}{|c|c|c|c|c|c|c|c|c|c|c|c|c|c|c|}
\hline & $\begin{array}{c}\text { Extremum point, } \\
\text { its localization }\end{array}$ & $m_{x x}$ & $m_{y y}$ & $m_{z z}$ & $R$ & $N_{v}$ & $\begin{array}{l}B_{m}^{2 D} / \\
B_{m, a b} \\
\end{array}$ & $\begin{array}{c}\text { Extremum point, } \\
\text { its localization }\end{array}$ & $m_{x x}$ & $m_{y y}$ & $m_{z z}$ & $R$ & $N_{v}$ & $\begin{array}{l}B_{m}^{2 D} / \\
B_{m, a b}\end{array}$ \\
\hline & \multicolumn{7}{|c|}{ Conduction band (CB), n-type doping } & \multicolumn{7}{|c|}{ Valence band (VB), p-type doping } \\
\hline $\mathrm{MoTe}_{2}$ & $\mathrm{~K}(1 / 3,1 / 3,0)$ & 0.52 & 0.52 & & & 2 & 4 & $\mathrm{~K}(1 / 3,1 / 3,0)$ & 0.63 & 0.65 & & & 2 & 4 \\
\hline \multirow[t]{2}{*}{$1 \mathrm{ML}$} & $\begin{array}{l}\Lambda(0.19,0.19,0) \\
\quad+0.005\end{array}$ & 0.35 & 0.29 & & & 6 & 36 & $\begin{array}{c}\Gamma(0,0,0) \\
-0.69\end{array}$ & 9.4 & 9.4 & & & 1 & 1 \\
\hline & & & & & & & & $\begin{array}{c}\mathrm{M}(1 / 2,0,0) \\
-0.13\end{array}$ & 0.82 & 0.90 & & & 3 & 9 \\
\hline $\mathrm{MoTe}_{2}$ & $\Lambda(0.2,0.2,0)$ & 0.36 & 0.32 & & & 6 & 36 & $\Gamma(0,0,0)$ & 11.16 & 11.73 & & & 1 & 1 \\
\hline \multirow[t]{2}{*}{$2 \mathrm{ML}$} & $\begin{array}{c}\mathrm{K}(1 / 3,1 / 3,0) \\
\quad+0.27\end{array}$ & 0.32 & 0.33 & & & 2 & 4 & $\begin{array}{c}\mathrm{K}(1 / 3,1 / 3,0) \\
\quad-0.16\end{array}$ & 0.62 & 1.57 & & & 2 & 4 \\
\hline & $\begin{array}{c}\mathrm{K}(1 / 3,1 / 3,0) \\
\quad+0.28\end{array}$ & 1.82 & 2.36 & & & 2 & 4 & $\begin{array}{c}\mathrm{M}(1 / 2,0,0) \\
-0.31\end{array}$ & 0.77 & 0.83 & & & 3 & 9 \\
\hline $\mathrm{MoTe}_{2}$ bulk & $\Lambda(0.2,0.2,0)$ & 0.38 & 0.33 & 0.44 & 1.2 & 6 & 21.1 & $\Gamma(0,0,0)$ & 0.6 & 0.6 & 2.77 & 4.6 & 1 & 1.7 \\
\hline \multirow[t]{4}{*}{$+2 \%$ strain } & $\begin{array}{c}\mathrm{K}(1 / 3,1 / 3,0) \\
\quad+0.13\end{array}$ & 1.62 & 3.10 & 8.47 & 3.8 & 2 & 5 & $\begin{array}{c}\mathrm{K}(1 / 3,1 / 3,0) \\
\quad-0.49\end{array}$ & 0.51 & 0.52 & 0.68 & 1.3 & 2 & 3.5 \\
\hline & $\begin{array}{l}\mathrm{K}(1 / 3,1 / 3,0) \\
\quad+0.14\end{array}$ & 0.25 & 0.25 & 15.88 & 63.5 & 2 & 12.7 & $\begin{array}{c}\mathrm{A}(0,0,1 / 2) \\
-0.09\end{array}$ & 1.41 & 1.41 & 0.04 & 0.03 & 1 & 0.3 \\
\hline & $\begin{array}{l}\mathrm{K}(1 / 3,1 / 3,0) \\
\quad+0.39\end{array}$ & 0.62 & 0.61 & 0.27 & 0.4 & 2 & 2.3 & & & & & & & \\
\hline & $\begin{array}{c}\mathrm{H}(1 / 3,1 / 3,1 / 2) \\
+0.15\end{array}$ & 0.42 & 0.42 & 1.16 & 2.8 & 2 & 4.5 & & & & & & & \\
\hline $\mathrm{MoTe}_{2}$ bulk & $\Lambda(0.2,0.2,0)$ & 0.31 & 0.27 & 0.34 & 1.2 & 6 & 21.1 & $\mathrm{M}(0,0,0)$ & 0.8 & 0.66 & 0.86 & 1.2 & 3 & 6.6 \\
\hline \multirow[t]{4}{*}{$-2 \%$ strain } & $\begin{array}{l}\mathrm{K}(1 / 3,1 / 3,0) \\
\quad+0.31\end{array}$ & 0.53 & 0.52 & 0.23 & 0.4 & 2 & 2.3 & $\begin{array}{c}\Gamma(0,0,0) \\
-0.17\end{array}$ & 1.48 & 1.48 & 1.23 & 0.8 & 1 & 0.9 \\
\hline & $\begin{array}{c}\mathrm{K}(1 / 3,1 / 3,0) \\
\quad+0.80\end{array}$ & 0.22 & 0.19 & 3.72 & 18.2 & 2 & 8.4 & $\begin{array}{c}\mathrm{K}(1 / 3,1 / 3,0) \\
-0.04\end{array}$ & 0.69 & 0.70 & 0.53 & 0.8 & 2 & 3 \\
\hline & $\begin{array}{c}\mathrm{H}(1 / 3,1 / 3,1 / 2) \\
+0.81\end{array}$ & 1.16 & 1.10 & 1.78 & 1.6 & 2 & 3.7 & & & & & & & \\
\hline & $\begin{array}{c}\mathrm{H}(1 / 3,1 / 3,1 / 2) \\
+0.81\end{array}$ & 1.16 & 1.10 & 1.78 & 1.6 & 2 & 3.7 & & & & & & & \\
\hline \multirow[t]{5}{*}{$\mathrm{MoTe}_{2}$ bulk } & $\Lambda(0.2,0.2,0)$ & 0.34 & 0.30 & 0.38 & 1.2 & 6 & 21.1 & $\Gamma(0,0,0)$ & 0.85 & 0.85 & 1.79 & 2.1 & 1 & 1.3 \\
\hline & $\begin{array}{l}\mathrm{K}(1 / 3,1 / 3,0) \\
\quad+0.34\end{array}$ & 0.57 & 0.56 & 0.25 & 0.4 & 2 & 2.3 & $\begin{array}{c}\mathrm{K}(1 / 3,1 / 3,0) \\
-0.21\end{array}$ & 0.59 & 0.61 & 0.57 & 1 & 2 & 3.2 \\
\hline & $\begin{array}{l}\mathrm{K}(1 / 3,1 / 3,0) \\
\quad+0.45\end{array}$ & 0.73 & 0.57 & 4.54 & 7 & 2 & 6.1 & $\begin{array}{c}\mathrm{A}(0,0,1 / 2) \\
-0.13\end{array}$ & 94.00 & 58.70 & 0.04 & 0.001 & 1 & 0.1 \\
\hline & $\begin{array}{l}\mathrm{K}(1 / 3,1 / 3,0) \\
\quad+0.47\end{array}$ & 0.21 & 0.22 & 9.83 & 45.7 & 2 & 11.4 & & & & & & & \\
\hline & $\begin{array}{c}\mathrm{H}(1 / 3,1 / 3,1 / 2) \\
+0.48\end{array}$ & 0.54 & 0.54 & 0.79 & 1.5 & 2 & 3.6 & & & & & & & \\
\hline
\end{tabular}



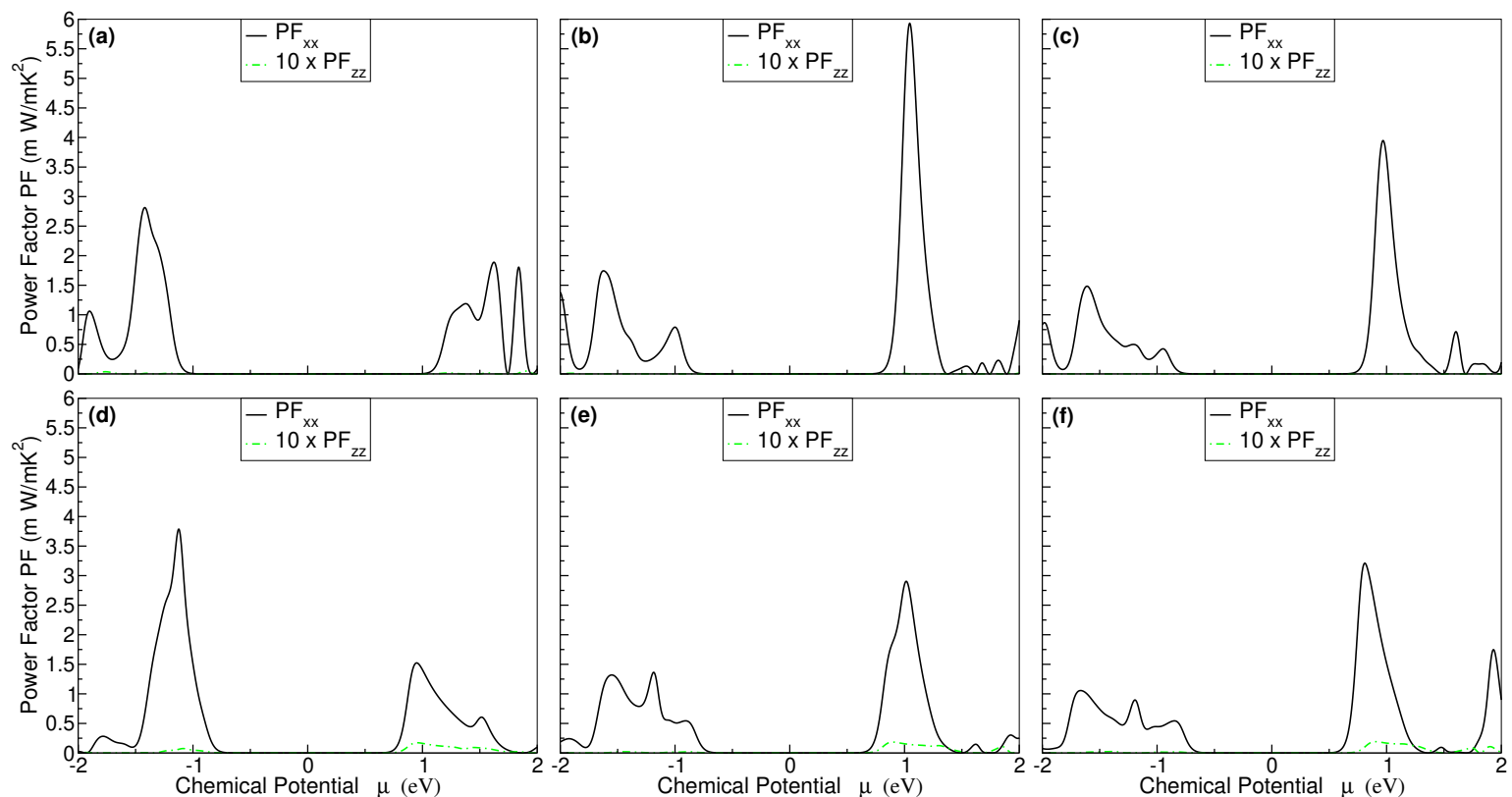

Figure S9: Power factor PF dependence on chemical potential for monolayers (ML) of WSe 2 (a) 1ML, (b) 2ML, (c) 3ML, and for (d) $\mathrm{WSe}_{2}-2 \%$ strained bulk, (e) $\mathrm{WSe}_{2}$ bulk, (f) $\mathrm{WSe}_{2}+1 \%$ strained bulk, estimated within B1-WC at $300 \mathrm{~K}$ using the relaxation time $\tau_{a}=1 \times 10^{-14} \mathrm{~s}$, and $\tau_{c}=1 \times 10^{-16} \mathrm{~s}$, respectively. $\mathrm{PF}_{x x}$ and $\mathrm{PF}_{z z}$ represent the components along $a$ and $c$ directions, $\mathrm{PF}_{z z}$ being scaled by a factor of 10 for a better comparison with $\mathrm{PF}_{x x}$.
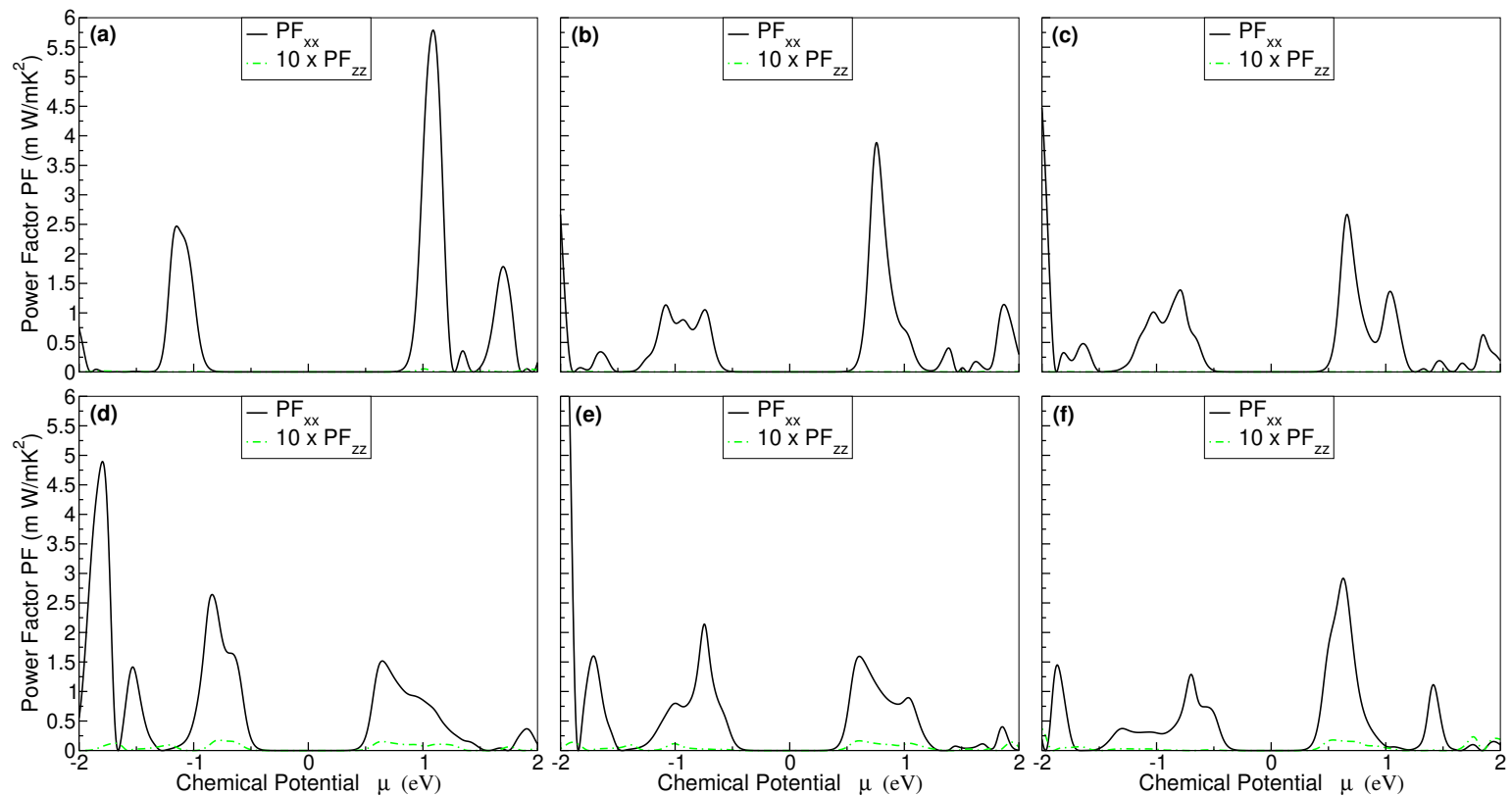

Figure S10: Power factor PF dependence on chemical potential for monolayers (ML) of MoTe 2 (a) 1ML, (b) 2ML, (c) 3ML, and for (d) $\mathrm{MoTe}_{2}-2 \%$ strained bulk, (e) $\mathrm{MoTe}_{2}$ bulk, (f) $\mathrm{MoTe}_{2}+2 \%$ strained bulk, estimated within B1-WC at $300 \mathrm{~K}$ using the relaxation time $\tau_{a}=1 \times 10^{-14} \mathrm{~s}$, and $\tau_{c}=1 \times 10^{-16} \mathrm{~s}$, respectively. $\mathrm{PF}_{x x}$ and $\mathrm{PF}_{z z}$ represent the components along $a$ and $c$ directions, $\mathrm{PF}_{z z}$ being scaled by a factor of 10 for a better comparison with $\mathrm{PF}_{x x}$. 

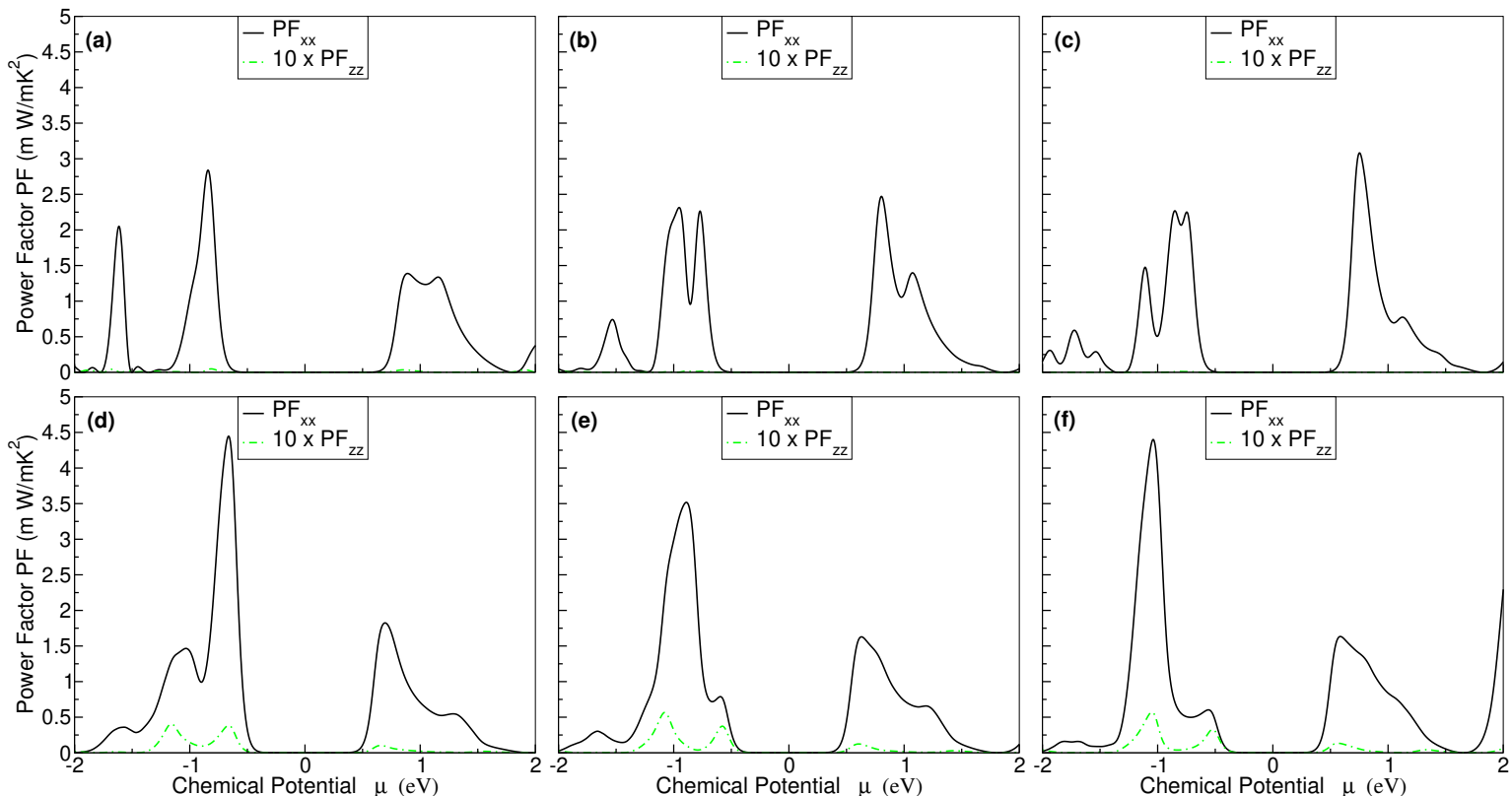

Figure S11: Power factor PF dependence on chemical potential for monolayers (ML) of SnSe 2 (a) 1ML, (b) 2ML, (c) 3ML, and for (d) $\mathrm{SnSe}_{2}-2 \%$ strained bulk, (e) $\mathrm{SnSe}_{2}$ bulk, (f) $\mathrm{SnSe}_{2} 2 \%$ strained bulk, estimated within B1-WC at $300 \mathrm{~K}$ using the relaxation time $\tau_{a}=1 \times 10^{-14} \mathrm{~s}$, and $\tau_{c}=1 \times 10^{-16} \mathrm{~s}$, respectively. $\mathrm{PF}_{x x}$ and $\mathrm{PF}_{z z}$ represent the components along $a$ and $c$ directions, $\mathrm{PF}_{z z}$ being scaled by a factor of 10 for a better comparison with $\mathrm{PF}_{x x}$. 

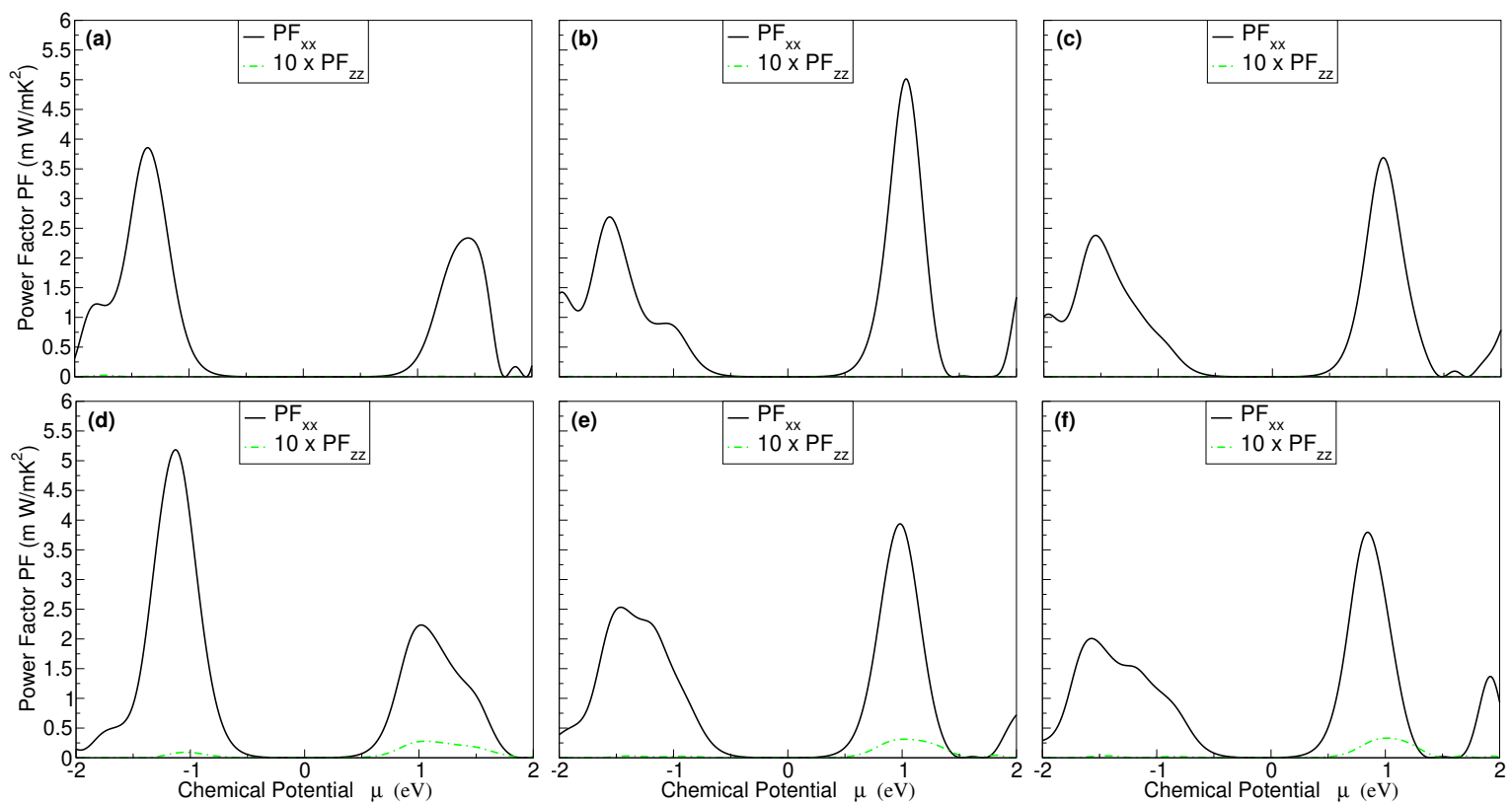

Figure S12: Power factor PF dependence on chemical potential for monolayers (ML) of WSe 2 (a) 1ML, (b) 2ML, (c) 3ML, and for (d) $\mathrm{WSe}_{2}-2 \%$ strained bulk, (e) $\mathrm{WSe}_{2}$ bulk, (f) $\mathrm{WSe}_{2}+1 \%$ strained bulk, estimated within B1-WC at $800 \mathrm{~K}$ using $1 / \mathrm{T}$ temperature dependence of the acoustic and optical relaxation times. $\mathrm{PF}_{x x}$ and $\mathrm{PF}_{z z}$ represent the components along $a$ and $c$ directions, $\mathrm{PF}_{z z}$ being scaled by a factor of 10 for a better comparison with $\mathrm{PF}_{x x}$.
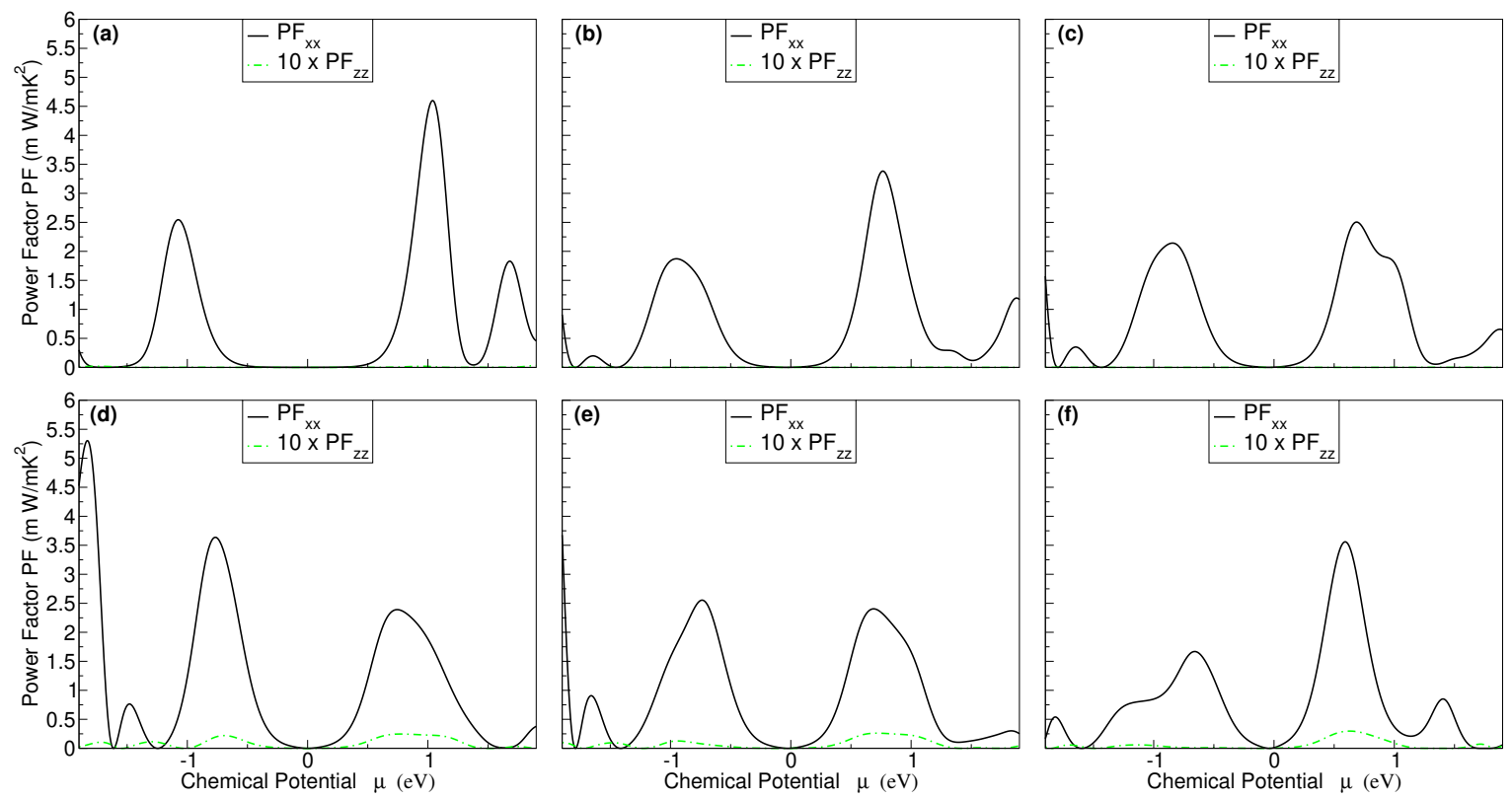

Figure S13: Power factor PF dependence on chemical potential for monolayers (ML) of MoTe 2 (a) 1ML, (b) 2ML, (c) 3ML, and for (d) $\mathrm{MoTe}_{2}-2 \%$ strained bulk, (e) $\mathrm{MoTe}_{2}$ bulk, (f) $\mathrm{MoTe}_{2}+2 \%$ strained bulk, estimated within B1-WC at $800 \mathrm{~K}$ using $1 / \mathrm{T}$ temperature dependence of the acoustic and optical relaxation times. $\mathrm{PF}_{x x}$ and $\mathrm{PF}_{z z}$ represent the components along $a$ and $c$ directions, $\mathrm{PF}_{z z}$ being scaled by a factor of 10 for a better comparison with $\mathrm{PF}_{x x}$. 

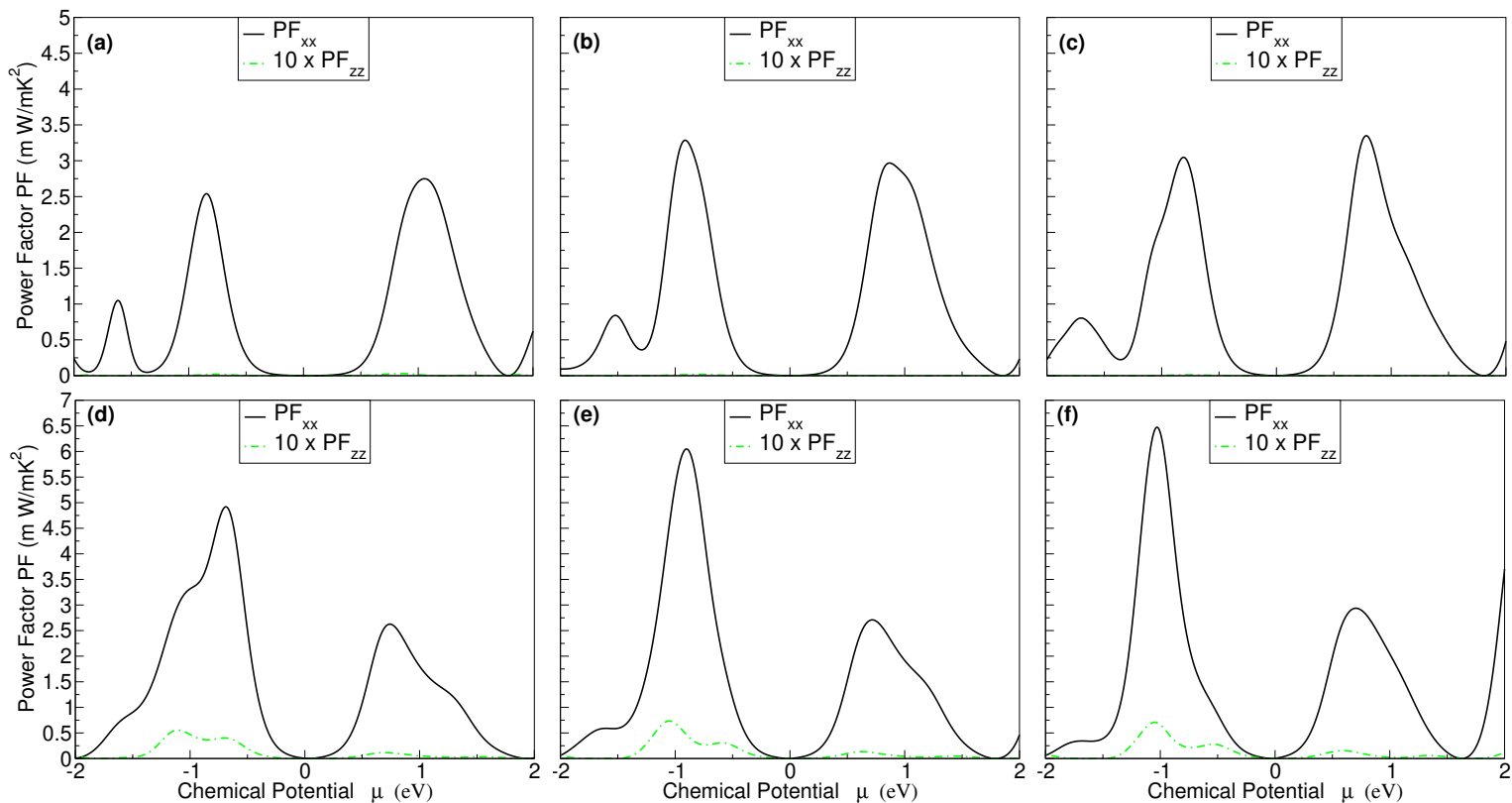

Figure S14: Power factor PF dependence on chemical potential for monolayers (ML) of SnSe 2 (a) 1ML, (b) 2ML, (c) 3ML, and for (d) $\mathrm{SnSe}_{2}-2 \%$ strained bulk, (e) $\mathrm{SnSe}_{2}$ bulk, (f) $\mathrm{SnSe}_{2} 2 \%$ strained bulk, estimated within B1-WC at $800 \mathrm{~K}$ using $1 / \mathrm{T}$ temperature dependence of the acoustic and optical relaxation times. $\mathrm{PF}_{x x}$ and $\mathrm{PF}_{z z}$ represent the components along $a$ and $c$ directions, $\mathrm{PF}_{z z}$ being scaled by a factor of 10 for a better comparison with $\mathrm{PF}_{x x}$. 

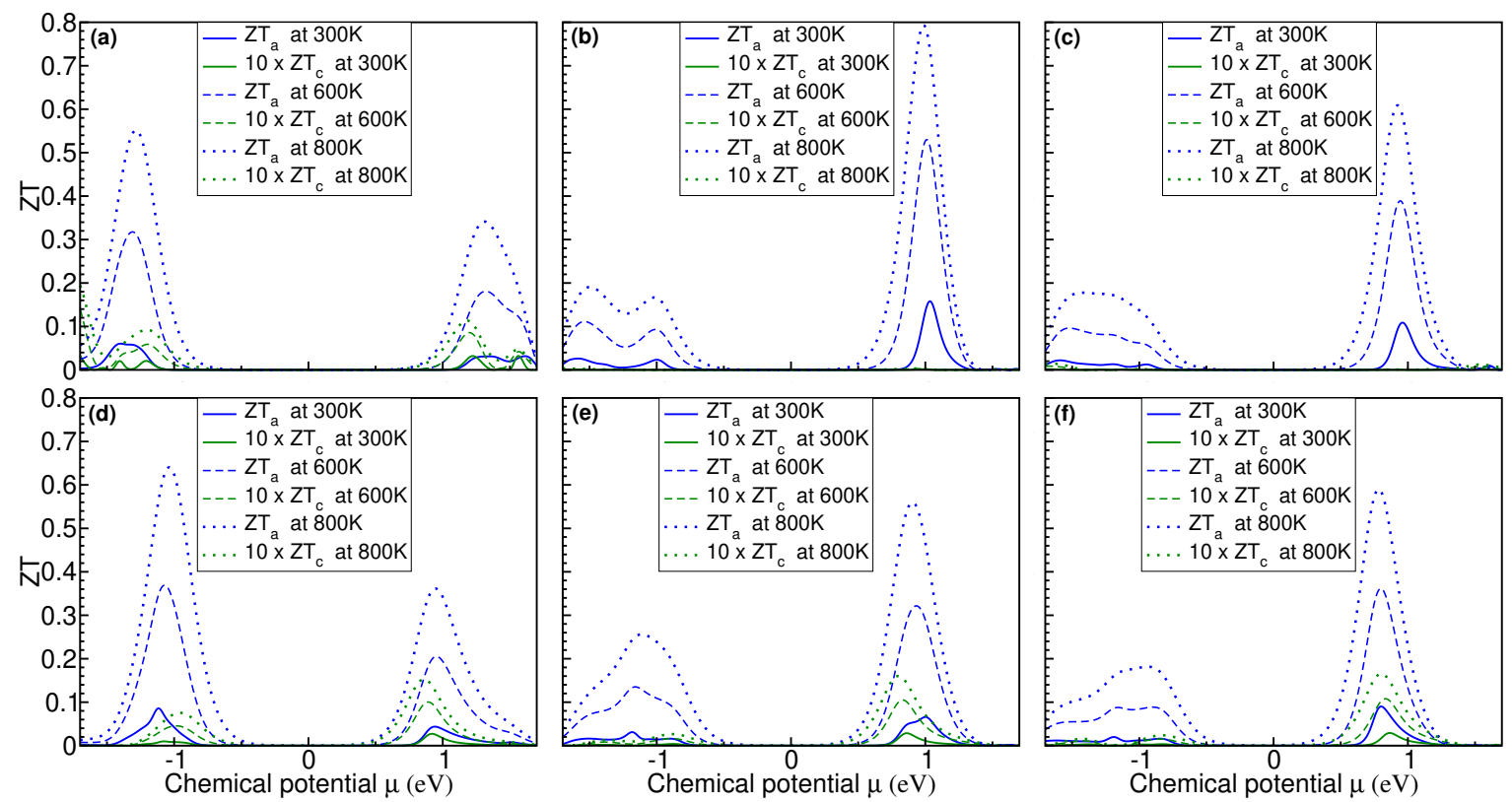

Figure S15: $\quad$ Figure of merit $Z T$ dependence on chemical potential $\mu$ for monolayers (ML) of WSe 2 (a) 1ML, (b) 2ML, (c) $3 \mathrm{ML}$, and for (d) $\mathrm{WSe}_{2}-2 \%$ strained bulk, (e) $\mathrm{WSe}_{2}$ bulk, (f) $\mathrm{WSe}_{2}+1 \%$ strained bulk, estimated within B1-WC at $300 \mathrm{~K}$, $600 \mathrm{~K}$, and $800 \mathrm{~K} . Z T_{a}$ and $Z T_{c}$ represent the components along $a$ and $c$ directions, $Z T_{c}$ being scaled by a factor of 10 for a better comparison with $Z T_{a}$.
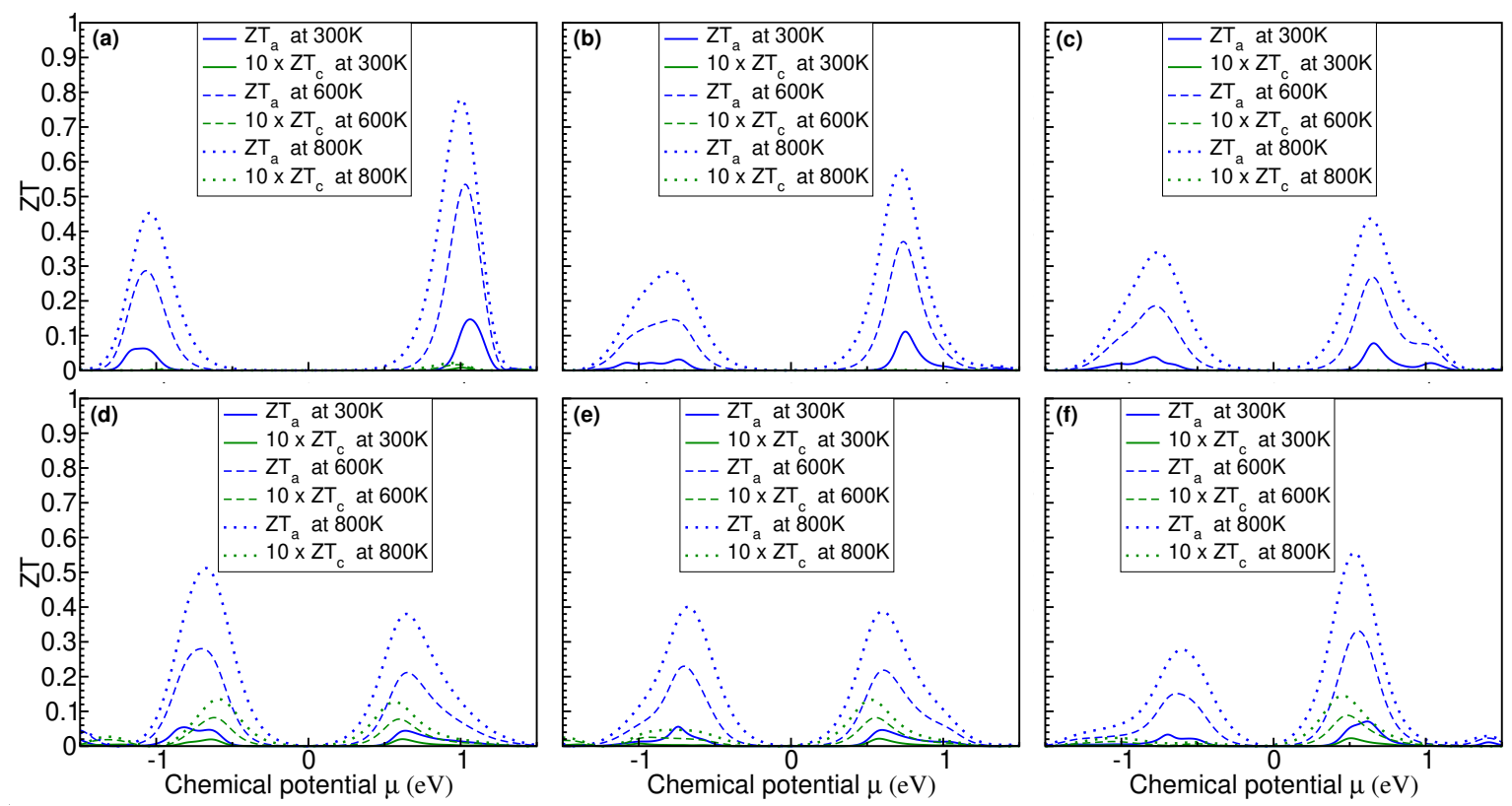

Figure S16: Figure of merit $Z T$ dependence on chemical potential $\mu$ for monolayers (ML) of MoTe 2 (a) $1 \mathrm{ML}$, (b) 2ML, (c) $3 \mathrm{ML}$, and for (d) $\mathrm{MoTe}_{2}-2 \%$ strained bulk, (e) $\mathrm{MoTe}_{2}$ bulk, (f) $\mathrm{MoTe}_{2}+2 \%$ strained bulk, estimated within B1-WC at 300 $\mathrm{K}, 600 \mathrm{~K}$, and $800 \mathrm{~K} . Z T_{a}$ and $Z T_{c}$ represent the components along $a$ and $c$ directions, $Z T_{c}$ being scaled by a factor of 10 for a better comparison with $Z T_{a}$. 

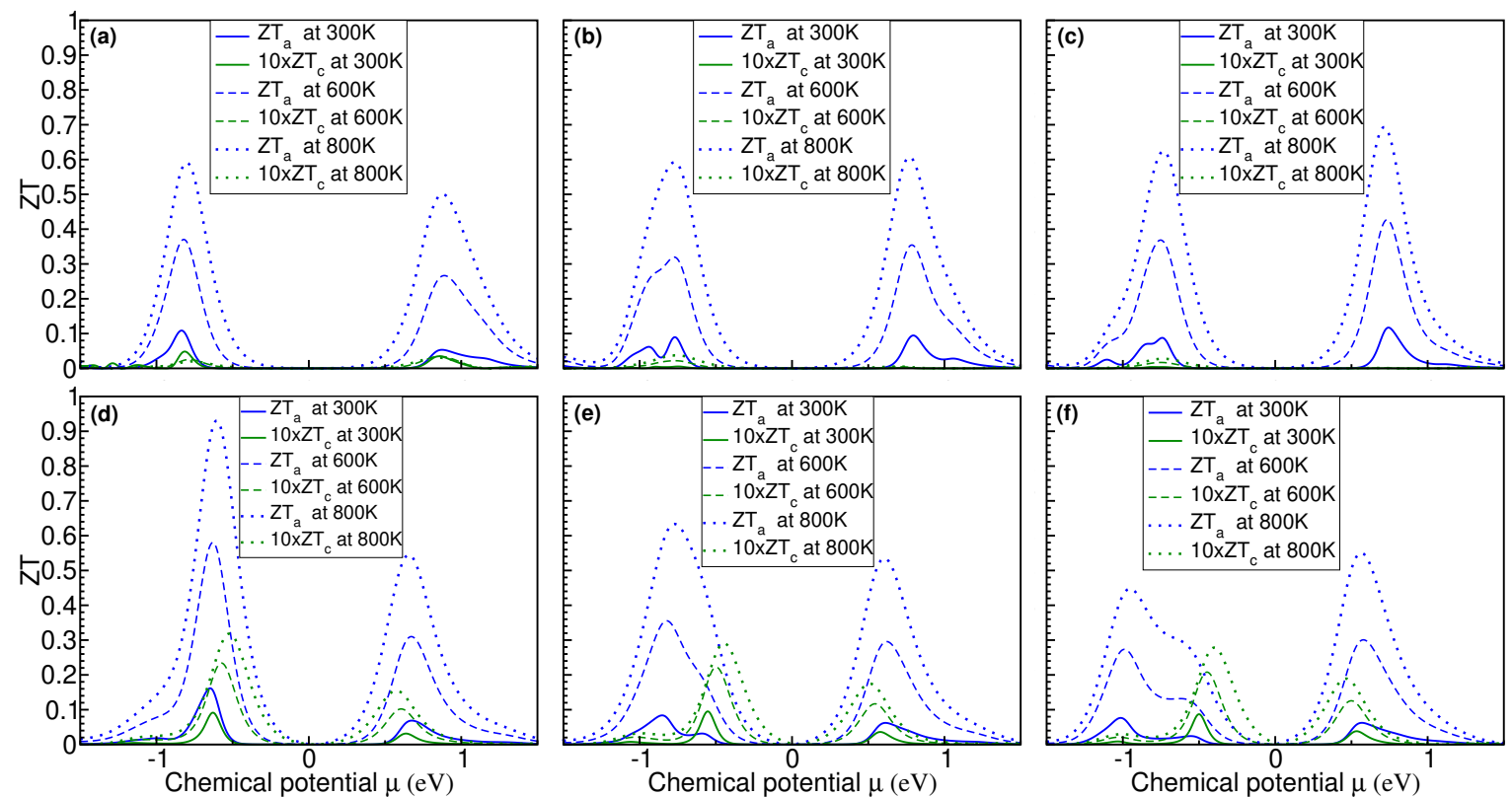

Figure S17: Figure of merit $Z T$ dependence on chemical potential $\mu$ for monolayers (ML) of SnSe 2 (a) 1ML, (b) 2ML, (c) $3 \mathrm{ML}$, and for (d) $\mathrm{SnSe}_{2}-2 \%$ strained bulk, (e) $\mathrm{SnSe}_{2}$ bulk, (f) $\mathrm{SnSe}_{2}+2 \%$ strained bulk, estimated within B1-WC at $300 \mathrm{~K}$, $600 \mathrm{~K}$, and $800 \mathrm{~K} . Z T_{a}$ and $Z T_{c}$ represent the components along $a$ and $c$ directions, $Z T_{c}$ being scaled by a factor of 10 for a better comparison with $Z T_{a}$.

\subsection{Explicit doping of TMDs}

We have checked if explicit doping of $\mathrm{Nb}$ at $\mathrm{W}$ site in $\mathrm{WSe}_{2}$ and $\mathrm{Sb}$ at $\mathrm{Sn}$ and Se sites in $\mathrm{SnSe}_{2}$ preserves the nonmagnetic character of $\mathrm{WSe}_{2}$ and $\mathrm{SnSe}_{2}$ doped semiconductors. We performed spin polarized calculations for $\mathrm{W}_{1-\frac{1}{16}} \mathrm{Nb}_{\frac{1}{16}} \mathrm{Se}_{2}$ using $4 \times 4 \times 1$ supercells, $\mathrm{Sn}_{1-\frac{1}{32}} \mathrm{Sb}_{\frac{1}{32}} \mathrm{Se}_{2}$ and $\mathrm{SnSe}_{2-\frac{1}{32}} \mathrm{Sb}_{\frac{1}{32}}$ using $4 \times 4 \times 2$ supercells allowing for nonmagnetic, ferromagnetic and antiferromagnetic orders on $\mathrm{Nb}$ and $\mathrm{Sb}$ doping elements and their nearest neighbours. From the spin up/down total density of states (DOS) of doped systems (Fig. S18), it can be seen the nonmagnetic character of $\mathrm{Nb}$ doped $\mathrm{WSe}_{2}$ and $\mathrm{Sb}$ doped $\mathrm{SnSe}_{2}$. $\mathrm{Nb}$ doping on $\mathrm{W}$ site acts as a p-type dopant in $\mathrm{WSe}_{2}$ (Fig. S18 a). Sb doping on Sn site acts as a p-type dopant in $\mathrm{SnSe}_{2}$ since $\mathrm{Sb}$ ion is in a $3+$ charging state $\left(\mathrm{Sb}^{3+}\right)$ comparing with the $4+$ charging state of $\mathrm{Sn}$ sublattice $\left(\mathrm{Sn}^{4+}\right)$, whereas $\mathrm{Sb}$ doping on Se site acts as a n-type dopant since $\mathrm{Sb}$ ion is in a 1- charging state $\left(\mathrm{Sb}^{1-}\right)$ comparing with the 2- charging state of Se sublattice $\left(\mathrm{Se}^{2-}\right)$ being in agreement with experimental results (Fig. $\mathrm{S} 18 \mathrm{~b}, \mathrm{c}) ! 18$
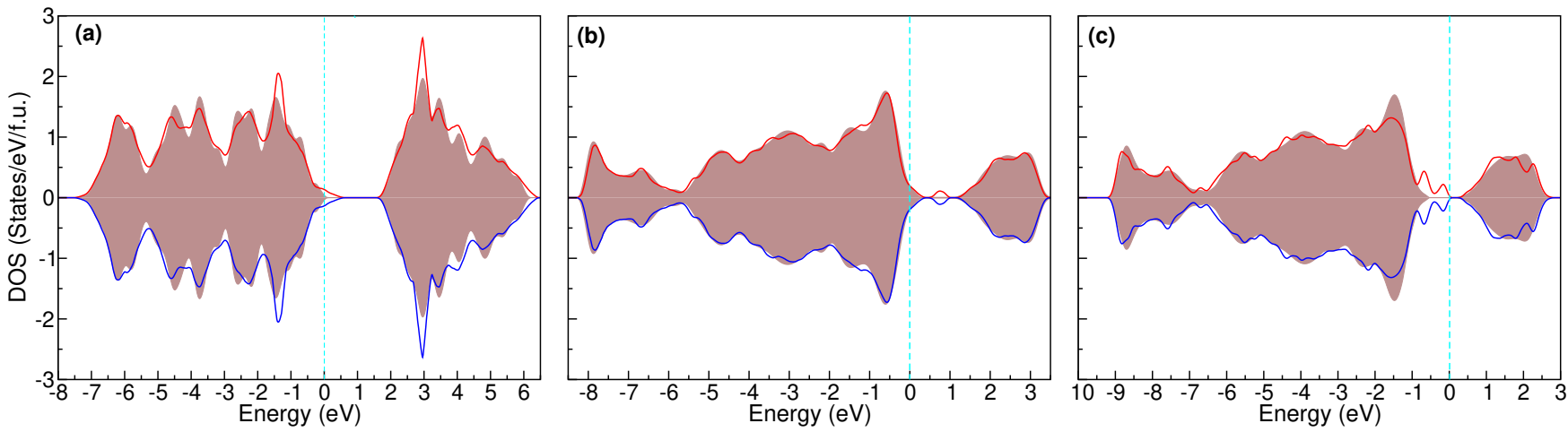

Figure S18: Spin up and spin down density of states (DOS) of: (a) $\mathrm{W}_{1-\frac{1}{16}} \mathrm{Nb}_{\frac{1}{16}} \mathrm{Se}_{2}$, (b) $\mathrm{Sn}_{1-\frac{1}{32}} \mathrm{Sb}_{\frac{1}{32}} \mathrm{Se}_{2}$ with $\mathrm{Sb}_{\text {ion }}$ in +3 charging state, $\mathrm{Sb}^{3+}$, and (c) $\mathrm{SnSe}_{2-\frac{1}{32}} \mathrm{Sb}_{\frac{1}{32}}$ with $\mathrm{Sb}$ ion in -1 charging state, $\mathrm{Sb}^{1-}$. Spin up/down DOS of WSe 2 and $\mathrm{SnSe}_{2}$ (f.u.) is shown in background brown colour for comparison. 
[1] Bilc, D. I.; Orlando, R.; Shaltaf, R.; Rignanese, G.M.; Iniguez, J.; Ghosez, Ph. Hybrid exchange-correlation functional for accurate prediction of the electronic and structural properties of ferroelectric oxides. Phys. Rev. B 2008, 77, 165107.

[2] Dovesi, R.; Erba, A.; Orlando, R.; Zicovich-Wilson, C. M.; Civalleri, B.; Maschio, L.; Rerat, M.; Casassa, S.; Baima, J.; Salustro, S.; et al. Quantum-mechanical condensed matter simulations with CRYSTAL. WIREs Comput. Mol. Sci. 2018, 8, e1360.

[3] Wu, Z.; Cohen, R. E. More accurate generalized gradient approximation for solids. Phys. Rev. B 2006, 73, 235116.

[4] Becke, A. D. Density-functional thermochemistry. IV. A new dynamical correlation functional and implications for exact-exchange mixing. J. Chem. Phys. 1996, 104, 1040-1046.

[5] Goffinet, M.; Hermet, P.; Bilc, D. I.; Ghosez, Ph. Hybrid functional study of prototypical multiferroic bismuth ferrite. Phys. Rev. B 2009, 79, 014403.

[6] Prikockyte, A.; Bilc, D.; Hermet, P.; Dubourdieu, C.; Ghosez, Ph. First-principles calculations of the structural and dynamical properties of ferroelectric $\mathrm{YMnO}_{3}$. Phys. Rev. B 2011, 84, 214301.

[7] Blaha, P.; Schwarz, K.; Tran, F.; Laskowski, R.; Madsen, G. K. H.; Marks, L. D. WIEN2k: an APW+lo program for calculating the properties of solids. J. Chem. Phys. 2020, 152, 074101.

[8] Steinberg, S.; Dronskowski, R. The crystal orbital Hamilton population (COHP) method as a tool to visualize and analyze chemical bonding in intermetallic compounds. Crystals 2018, 8, 225.

[9] Hicks, L. D.; Dresselhaus, M. S. Effect of quantum-well structures on the thermoelectric figure of merit. Phys. Rev. B 1993, 47, 12727.
[10] Pei, Y.; LaLonde, A. D.; Wang, H.; Snyder, G. J. Low effective mass leading to high thermoelectric performance. Energy Environ. Sci. 2012, 5, 7963-7969.

[11] Zhang, X.; Bu, Z.; Shi, X.; Chen, Z.; Lin, S.; Shan, B.; Wood, M.; Snyder, A. H.; Chen, L.; Snyder, G. F.; et al. Electronic quality factor for thermoelectrics. Sci. Adv. 2020, 6, eabc0726.

[12] Bardeen, J.; Shockley, W. Deformation potentials and mobilities in non-polar crystals. Phys. Rev. 1950, 80, 72.

[13] Herring, C.; Vogt, E. Transport and deformationpotential theory for many-valley semiconductors with anisotropic scattering. Phys. Rev. 1956, 101, 944.

[14] Gantmakher, V. F.; Levinson, Y. B. In Carrier Scattering in Metals and Semiconductors; Agranovich, V. M., Maradudin, A. A., Eds.; Elsevier-North-Holland, 1987; Vol. 19 of Modern Problems in Condensed Matter Sciences.

[15] Parker, D.; May, A. F.; Singh, D. J. Benefits of carrier pocket anisotropy to thermoelectric performance: the case of p-type $\mathrm{AgBiSe}_{2}$. Phys. Rev. Appl. 2015, 3, 064003.

[16] Gibbs, Z. M.; Ricci, F.; Li, G.; Zhu, H.; Persson, K.; Ceder, G.; Hautier, G.; Jain, A.; Snyder, G. J. Effective mass and Fermi surface complexity factor from abinitio band structure calculations. npj Comput. Mater. 2017, $3,8$.

[17] Price, P. J. Two-dimensional electron transport in semiconductor layers. Ann. Phys. 1981, 133, 217-239.

[18] Choe, J. S.; Lee, C.; Kim, M. J.; Lee, G.-G.; Shim, J.-H.; Lim, Y. S. Bader net charge analysis on doping effects of $\mathrm{Sb}$ in $\mathrm{SnSe}_{2}$ and related charge transport properties. $J$. Appl. Phys. 2020, 127, 185706. 